\title{
A QUEUE WITH SEMIPERIODIC TRAFFIC
}

\author{
JUAN ALVAREZ *** AND \\ BRUCE HAJEK, ${ }^{* * * *}$ University of Illinois at Urbana-Champaign
}

\begin{abstract}
In this paper, we analyze the diffusion limit of a discrete-time queueing system with constant service rate and connections that randomly enter and depart from the system. Each connection generates periodic traffic while it is active, and a connection's lifetime has finite mean. This can model a time division multiple access system with constant bit-rate connections. The diffusion scaling retains semiperiodic behavior in the limit, allowing for both short-time analysis (within one frame) and long-time analysis (over multiple frames). Weak convergence of the cumulative arrival process and the stationary buffer-length distribution is proved. It is shown that the limit of the cumulative arrival process can be viewed as a discrete-time stationary-increment Gaussian process interpolated by Brownian bridges. We present bounds on the overflow probability of the limit queueing process as functions of the arrival rate and the connection lifetime distribution. Also, numerical and simulation results are presented for geometrically distributed connection lifetimes.
\end{abstract}

Keywords: Semiperiodic traffic; diffusion limit; overflow; Gaussian process; queueing

2000 Mathematics Subject Classification: Primary 60G15

Secondary 60F99; 60G35

\section{Introduction}

Some types of real-time traffic sources, like digitized voice, generate data in a regular, periodic fashion. In this paper, we consider such traffic in a FIFO (first-in-first-out) queueing system within the heavy-traffic regime, where diffusion limits can be used to simplify the analysis.

Consider a time-slotted queueing system with constant service rate. Each connection enters the system, generates one packet every $N$ slots (for some positive integer $N$ ), and departs after transmitting a random number of packets, independently of the other connections and of the time slot during which the connection began generating packets. Packets are queued for transmission, and the transmission time of each packet is one slot. The numbers of new connections in distinct slots are assumed to be mutually independent Poisson random variables. We assume that the connection lifetimes are identically distributed with finite mean.

We are interested in analyzing the performance of this system. This is a rather complex task, so we look at the diffusion limit of the system in order to simplify the analysis and obtain an estimate of its behavior. We find a limit of the scaled cumulative arrival process as $N \rightarrow \infty$, uniformly over the scaled arrival rate. The semiperiodic nature of the network is reflected in the

Received 17 June 2002; revision received 28 October 2004.

* Postal address: Coordinated Science Laboratory, University of Illinois at Urbana-Champaign, 1308 West Main Street, Urbana, IL 61801, USA.

** Email address: alvarez@comm.csl.uiuc.edu

*** Email address: b-hajek@uiuc.edu

Supported by the National Science Foundation under grant NSF ANR 99-80544. 
form of the autocorrelation function of the limit process. We show that the limit can be viewed as a discrete-time stationary-increment Gaussian process interpolated by Brownian bridges, and we present bounds on the overflow probability of the limit queueing process as functions of the arrival rate and the connection lifetime distribution.

Our results complement those of Addie et al. [1] and Norros [8], who applied large-deviation techniques to the analysis of queues with stationary Gaussian arrival processes. In particular, the limit process in this paper is considered as an example in [8]. Similar models are considered in Hajek [5] and Pazhyannur and Fleming [9]. In [5], the number of connections is fixed for each $N$, as $N \rightarrow \infty$, while, in [9], a heavy-traffic limit for fixed $N$ is considered for a model with a Gaussian arrival process.

The paper is organized as follows. In Section 2, we present the system model, the distribution of packet arrivals, and the buffering process. The proof of the convergence of the arrival process is carried out in Section 3 and, in Section 4, we establish some properties of the limit process. In Section 5, we establish convergence of the normalized buffer-length distribution and, in Section 6, present bounds on the overflow probability of the limit queueing system. Finally, in Section 7, we present numerical and simulation results for geometrically distributed connection lifetimes.

\section{The system model}

In this section, we present the model to be used throughout the paper. For integer $m$, slot $m$ represents the time interval $[m, m+1)$. We group $N$ consecutive slots together and call this a frame. The set of all slots is divided into $N$ equivalence classes, referred to as phases: slots $m$ and $l$ are in the same phase if $m=l+n N$ for some integer $n$. Therefore, each frame consists of one slot from each of the $N$ phases. Figure 1 depicts the relationships among frames, phases, slots, and continuous time.

Let $L$ denote the number of packets sent by a connection (referred to as connection lifetime throughout the rest of the paper), let its probability mass function be denoted by $f_{L}(l)$, for $l \geq 1$, and let it have mean $\bar{L}<\infty$. Also, let $F_{L}^{\mathrm{c}}(l):=\mathrm{P}[L \geq l]$. The connection lifetimes are mutually independent.

Let $x_{k, j}$ denote the number of new connections in phase $k$ of frame $j$, for $k=0, \ldots, N-1$ and $j \geq 0$, and let $0<\lambda_{\min } \leq \bar{L}^{-1}$. Assume that $x_{k, j}$ has the Poisson distribution with mean $\lambda_{N}$ such that $\lambda_{N} \in\left[\lambda_{\min }, \bar{L}^{-1}\right]$. The numbers of new connections in different slots are mutually independent. Since a connection generates one packet every $N$ slots, if it enters the system during phase $k$ of a frame, then it will keep sending one packet every frame (in the same phase) for a random number of frames. Clearly, the packet arrivals in different phases are independent of each other due to the independence of the connections.

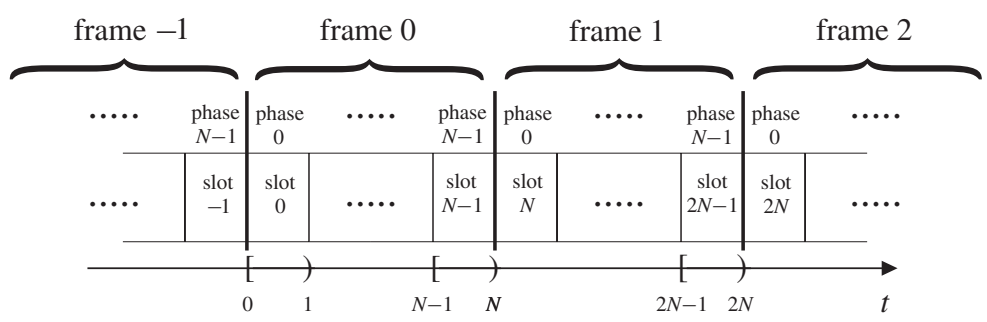

FIGURE 1: The system model. 
We denote the number of packets that arrive in slot $m$ by $\tilde{a}_{m}$, and by $a_{k, j}$ the number of packets that arrive in phase $k$ of frame $j$. This implies that $a_{k, j}=\tilde{a}_{k+N j}$. We assume that packet arrivals occur only at the beginning of each slot.

Let $m_{1}$ and $m_{2}$ be integers with $m_{1} \leq m_{2}$. A connection is said to have endpoints $m_{1}$ and $m_{2}$ if its first packet is generated in slot $m_{1}$ and its last packet is generated in slot $m_{2}$. If $m_{2}-m_{1}=N(l-1)$ for some positive integer $l$, then the number of connections with endpoints $m_{1}$ and $m_{2}$ has the Poisson distribution with mean $\lambda_{N} f(l)$. Otherwise, there are no connections with endpoints $m_{1}$ and $m_{2}$. From this observation, it is clear that the arrival process $\left(\tilde{a}_{m}\right)$ is time reversible.

Next, in Section 2.1, we give the distribution of the number of packets arriving into the system in any particular slot. Section 2.2 concerns the buffering process.

\subsection{The distribution of packet arrivals}

Since arrivals in different phases are independent, and the distribution of arrivals within each phase is the same, it suffices to obtain the distribution of arrivals within one phase.

Theorem 1. For any $k=0, \ldots, N-1$ and integer $j$, the distribution of $a_{k, j}$ is Poisson with mean $\lambda_{N} \bar{L}$. Furthermore, if $j_{1}, j_{2}, k_{1}$, and $k_{2}$ are integers with $0 \leq k_{1}, k_{2} \leq N-1$, then

$$
\operatorname{cov}\left(a_{k_{1}, j_{1}}, a_{k_{2}, j_{2}}\right)=\lambda_{N} \bar{L} g\left(\left|j_{1}-j_{2}\right|+1\right) \delta_{k_{1}, k_{2}},
$$

where $g(l):=\bar{L}^{-1} \sum_{n=l}^{\infty} F_{L}^{\mathrm{c}}(n)$ and $\delta$ is the Kronecker delta function.

Proof. Fix $k$ and let $j \in \mathbb{Z}$. Then the number of packet arrivals in phase $k$ of frame $j$ corresponds to the number of active connections in the same frame and phase. This can be considered to be the number of busy servers in an $\mathrm{M} / \mathrm{G} / \infty$ queue with Poisson arrivals with mean $\lambda_{N}$ and independent, identically distributed service times, with probability mass function $f_{L}$. Therefore, its steady-state distribution has the following properties [7]: $a_{k, j} \sim \operatorname{Poisson}\left(\lambda_{N} \bar{L}\right)$ and

$$
\operatorname{cov}\left(a_{k, j}, a_{k, j+n}\right)=\lambda_{N} \mathrm{E}\left[(L-|n|)^{+}\right]=\lambda_{N} \sum_{l=|n|+1}^{\infty} F_{L}^{\mathrm{c}}(l),
$$

where $x^{+}$denotes the positive part of $x$.

If $k_{1} \neq k_{2}$ then, by independence between phases, $\operatorname{cov}\left(a_{k_{1}, j}, a_{k_{2}, j+n}\right)=0$. Hence, the proposition is proved.

In Section 3, we will use these properties of the arrival process in order to obtain a diffusion limit for the model. It was pointed out in [7] that the process could be long-range dependent. In particular, if $\mathrm{E}\left[L^{2}\right]=\infty$ then $\operatorname{cov}\left(a_{k, j}, a_{k, j+n}\right)$ is not summable in $n$.

\subsection{Buffer size}

In this section, we consider the buffering process. Let $B_{m}$ be the cumulative number of arrivals from slot $-m$ up to slot -1 , i.e. $B_{m}=\sum_{l=-m}^{-1} \tilde{a}_{l}$. Then the buffer size at time 0 , denoted by $Q_{0}^{N}$, can be expressed as $Q_{0}^{N}=\sup \left\{0, B_{1}-1, B_{2}-2, \ldots\right\}$.

Let $\tilde{A}_{m}$ denote the cumulative number of arrivals in $[0, m)$, i.e.

$$
\tilde{A}_{m}= \begin{cases}\sum_{l=0}^{m-1} \tilde{a}_{l}, & m \geq 1, \\ 0, & m=0 .\end{cases}
$$


Then, by the reversibility of the arrival process,

$$
Q_{0}^{N} \stackrel{\mathrm{D}}{=} \sup \left\{0, \tilde{A}_{1}-1, \tilde{A}_{2}-2, \ldots\right\}=\sup _{m \geq 0}\left\{\tilde{A}_{m}-m\right\}
$$

where ' $=$, denotes equality in distribution.

It will be helpful to group the arrivals by phases, so we denote by $A_{k, j}$ the cumulative number of phase- $k$ arrivals in frames $\{0,1, \ldots, j-1\}$, i.e. for $k=0, \ldots, N-1$,

$$
A_{k, j}= \begin{cases}\sum_{l=0}^{j-1} a_{k, l}, & j \geq 1 \\ 0, & j=0 .\end{cases}
$$

Using (4) and the fact that packet arrivals occur only at the beginning of each slot, we can rewrite (2) as

$$
\tilde{A}_{t}=\sum_{k=0}^{N-1} A_{k, l_{k}^{N}(t)} \text { for all } t \geq 0
$$

where $l_{k}^{N}(t)=\lfloor t+1-(1+k) / N\rfloor$, i.e. $l_{k}^{N}(t)$ denotes the number of complete phase- $k$ slots in $[0, t]$ (since $\lfloor\cdot\rfloor$ denotes the largest integer less than or equal to its argument). Notice that this definition of $l_{k}^{N}(t)$ allows us to use any nonnegative real number $t$, instead of just integer values.

We now consider a diffusion limit of this cumulative arrival process, in Section 3.

\section{The diffusion limit}

In this section, we present a diffusion limit for our model that allows for two time-scales: a short one, corresponding to a single frame, and a long one, which corresponds to multiple frames. For $t \geq 0$ and for $N \in \mathbb{N}$, we define

$$
X_{t}^{N}=\frac{\tilde{A}_{N t}-\lambda_{N} \bar{L}\lfloor N t\rfloor}{\sqrt{\lambda_{N} \bar{L} N}} .
$$

For any $T>0$, the process $X_{t}^{N}$ is a member of $D[0, T]$, the space of right-continuous functions with left limits on the interval $[0, T]$. We denote by ' $\stackrel{\mathrm{W}}{\rightarrow}$ ' weak convergence with respect to the Skorokhod topology [2] on $D[0, T]$.

Let $\left\{X_{t}\right\}_{t \geq 0}$ be a stationary-increment, zero-mean, almost-surely-continuous Gaussian process with variance function

$$
\rho_{t}:=\mathrm{E}\left[X_{t}^{2}\right]=t+2 \sum_{j=1}^{\infty} g(j+1)(t-j)^{+} \quad \text { for all } t \geq 0
$$

Notice that the variance is piecewise linear with slope $1+2 \sum_{\{j: 1 \leq j \leq k\}} g(j+1)$ on the interval $[k, k+1]$, where $k$ is a nonnegative integer. This slope approaches $(2 / \bar{L}) \mathrm{E}\left[L^{2}\right]-1$ 
as $k \rightarrow \infty$ and, as indicated in Section 2.1, if $\mathrm{E}\left[L^{2}\right]=\infty$ then the process is long-range dependent.

The covariance function of $X$ is obtained from its stationary-increments property and its variance, i.e.

$$
\rho_{s, t}:=\mathrm{E}\left[X_{s} X_{t}\right]=\frac{1}{2}\left(\rho_{s}+\rho_{t}-\rho_{|s-t|}\right) .
$$

Since $\rho_{t}=t$ for $0 \leq t \leq 1$, the restriction $\left\{X_{t}\right\}_{0 \leq t \leq 1}$ is a standard Brownian motion. Since $X$ has stationary increments, $\left\{X_{t+\alpha}-X_{\alpha}\right\}_{0 \leq t \leq 1}$ is also a standard Brownian motion for any fixed $\alpha \geq 0$. Additional properties of $X$ are given in Section 4 .

Theorem 2. For each $T>0$, the random process $\left\{X_{t}^{N}\right\}_{0 \leq t \leq T}$ converges weakly to the random process $\left\{X_{t}\right\}_{0 \leq t \leq T}$ in $D[0, T]$ as $N \rightarrow \infty$, i.e. $X^{N} \stackrel{\mathrm{W}}{\rightarrow} X$.

Proof. The proof is carried out in three steps. First, convergence of one-dimensional distributions is shown. This result is then extended to finite-dimensional distributions and, finally, to weak convergence with respect to the Skorokhod topology on $D[0, T]$.

Step 1. Let $t \geq 0$ be fixed throughout this step of the proof. We can rewrite (5) as

$$
X_{t}^{N}=\frac{1}{\sqrt{\lambda_{N} \bar{L} N}} \sum_{k=0}^{N-1} S_{k}^{N}(t),
$$

where

$$
S_{k}^{N}(t)=A_{k, l_{k}^{N}(N t)}-l_{k}^{N}(N t) \lambda_{N} \bar{L},
$$

i.e. $S_{k}^{N}(t)$ is the cumulative number of arrivals in phase $k$ up to time $N t$, minus its mean.

Clearly, for fixed $t$, the random variables $S_{k}^{N}(t), k=0, \ldots, N-1$, are mutually independent since they correspond to arrivals in different phases. This representation of $X_{t}^{N}$ makes it evident that it is the sum of $N$ independent random variables.

The first and second moments of $S_{k}^{N}(t)$, as well as an upper bound on its third moment, will be useful to show the desired convergence. The process $S_{k}^{N}$ is centered so that $\mathrm{E}\left[S_{k}^{N}(t)\right]=0$. The second moment is given by

$$
\begin{aligned}
\mathrm{E}\left[S_{k}^{N}(t)^{2}\right] & =\sum_{j_{1}=0}^{l_{k}^{N}(N t)-1} \sum_{j_{2}=0}^{l_{k}^{N}(N t)-1} \operatorname{cov}\left(a_{k, j_{1}}, a_{k, j_{2}}\right) \\
& =\sum_{j=0}^{l_{k}^{N}(N t)-1} \operatorname{var}\left(a_{k, j}\right)+2 \sum_{j=1}^{l_{k}^{N}(N t)-1}\left(l_{k}^{N}(N t)-j\right) \operatorname{cov}\left(a_{k, 0}, a_{k, j}\right) \\
& =l_{k}^{N}(N t) \lambda_{N} \bar{L}+2 \sum_{j=1}^{l_{k}^{N}(N t)-1}\left(l_{k}^{N}(N t)-j\right) \lambda_{N} \bar{L} g(j+1) \\
& = \begin{cases}\lambda_{N} \bar{L}\left(\lfloor t\rfloor+1+2 \sum_{j=1}^{\lfloor t\rfloor}(\lfloor t\rfloor+1-j) g(j+1)\right) & \text { if } t-\lfloor t\rfloor \geq \frac{k+1}{N}, \\
\lambda_{N} \bar{L}\left(\lfloor t\rfloor+2 \sum_{j=1}^{\lfloor t\rfloor}(\lfloor t\rfloor-j) g(j+1)\right) & \text { otherwise, }\end{cases}
\end{aligned}
$$


where the second equality follows from the stationarity of the arrivals, the third follows from (1), and the fourth follows from the fact that $l_{k}^{N}(N t)=\lfloor t\rfloor+1$ if $t-\lfloor t\rfloor \geq(k+1) / N$ and $l_{k}^{N}(N t)=\lfloor t\rfloor$ otherwise. Note that if $a, b \geq 0$, then $|a-b|^{3} \leq \max \left\{a^{3}, b^{3}\right\} \leq a^{3}+b^{3}$. Thus,

$$
\begin{aligned}
\mathrm{E}\left[\left|S_{k}^{N}(t)\right|^{3}\right] & \leq \mathrm{E}\left[\left(\sum_{m=1}^{l_{k}^{N}(N t)} m Z_{m}\right)^{3}+\left(\sum_{m=1}^{l_{k}^{N}(N t)} m \mu_{m}\right)^{3}\right] \\
& \leq\left(l_{k}^{N}(N t)\right)^{3} \mathrm{E}\left[\left(\sum_{m=1}^{l_{k}^{N}(N t)} Z_{m}\right)^{3}+\left(\sum_{m=1}^{l_{k}^{N}(N t)} \mu_{m}\right)^{3}\right] \\
& \leq\left(l_{k}^{N}(N t)\right)^{3}\left(\left(1+\sum_{m=1}^{l_{k}^{N}(N t)} \mu_{m}\right)^{3}+\left(\sum_{m=1}^{l_{k}^{N}(N t)} \mu_{m}\right)^{3}\right) \\
& \leq\left(l_{k}^{N}(N t)\right)^{3}\left(1+2 \sum_{m=1}^{l_{k}^{N}(N t)} \mu_{m}\right)^{3} \\
& \leq\left(l_{k}^{N}(N t)\right)^{3}\left(1+2 \lambda_{N} \bar{L} l_{k}^{N}(N t)\right)^{3} \\
& \leq\lceil t\rceil^{3}\left(1+2 \lambda_{N} \bar{L}\lceil t\rceil\right)^{3},
\end{aligned}
$$

where $Z_{m}$ is the number of connections that contribute $m$ arrivals to $S_{k}^{N}(t), \mu_{m}=\mathrm{E}\left[Z_{m}\right]$, and $\lceil\cdot\rceil$ denotes the smallest integer greater than or equal to its argument. The $Z_{m}$ are mutually independent Poisson random variables and $\sum_{m=1}^{l_{k}^{N}(N t)} m \mu_{m}=\lambda_{N} \bar{L} l_{k}^{N}(N t)$. The first inequality follows because both $Z_{m}$ and $\mu_{m}$ are nonnegative; the third follows because, for a Poisson random variable with mean $\mu$, its third moment equals $\mu^{3}+3 \mu^{2}+\mu<(1+\mu)^{3}$; and the final one follows because $l_{k}^{N}(N t) \leq\lceil t\rceil$.

It will now be shown that the random variable $X_{t}^{N}$ converges in distribution to a Gaussian random variable. For each integer $N \geq 1$ and $k=0, \ldots, N-1$, let $Y_{N, k}=\left(\lambda_{N} \bar{L} N\right)^{-1 / 2} S_{k}^{N}(t)$. Then $\mathrm{E}\left[Y_{N, k}\right]=0$ and

$$
\begin{aligned}
\sum_{k=0}^{N-1} \mathrm{E}\left[Y_{N, k}^{2}\right]= & \frac{1}{\lambda_{N} \bar{L} N} \sum_{k=0}^{N-1} \mathrm{E}\left[S_{k}^{N}(t)^{2}\right] \\
= & \frac{\lfloor N t\rfloor-N\lfloor t\rfloor}{N}\left(\lfloor t\rfloor+1+2 \sum_{j=1}^{\lfloor t\rfloor}(\lfloor t\rfloor+1-j) g(j+1)\right) \\
& +\frac{N-(\lfloor N t\rfloor-N\lfloor t\rfloor)}{N}\left(\lfloor t\rfloor+2 \sum_{j=1}^{\lfloor t\rfloor-1}(\lfloor t\rfloor-j) g(j+1)\right) \\
= & \frac{\lfloor N t\rfloor}{N}+2 \sum_{j=1}^{\lfloor t\rfloor}\left(\frac{\lfloor N t\rfloor}{N}-j\right) g(j+1) \\
\rightarrow & t+2 \sum_{j=1}^{\lfloor t\rfloor}(t-j) g(j+1) \\
= & \rho_{t},
\end{aligned}
$$


where the second equality follows from (8) and the fact that $t-\lfloor t\rfloor \geq(k+1) / N$ for the first $\lfloor N t\rfloor-N\lfloor t\rfloor$ terms in the sum. The third equality follows from grouping terms. Notice that $\rho_{t}<\infty$.

Now, for any $\varepsilon>0$,

$$
\begin{aligned}
\sum_{k=0}^{N-1} \mathrm{E}\left[\left|Y_{N, k}\right|^{2} ;\left|Y_{N, k}\right|>\varepsilon\right] & \leq \sum_{k=0}^{N-1} \mathrm{E}\left[\frac{\left|Y_{N, k}\right|^{3}}{\varepsilon}\right]=\frac{1}{\varepsilon\left(\lambda_{N} \bar{L} N\right)^{3 / 2}} \sum_{k=0}^{N-1} \mathrm{E}\left[\left|S_{k}^{N}(t)\right|^{3}\right] \\
& \leq \frac{1}{\varepsilon\left(\lambda_{N} \bar{L} N\right)^{3 / 2}} \sum_{k=0}^{N-1}\lceil t\rceil^{3}\left(1+2 \lambda_{N} \bar{L}\lceil t\rceil\right)^{3} \\
& \leq N \frac{\lceil t\rceil^{3}(1+2\lceil t\rceil)^{3}}{\varepsilon\left(\lambda_{N} \bar{L} N\right)^{3 / 2}} \leq \frac{\lceil t\rceil^{3}(1+2\lceil t\rceil)^{3}}{\varepsilon\left(\lambda_{\min } \bar{L}\right)^{3 / 2}} \frac{1}{\sqrt{N}} \\
& \rightarrow 0,
\end{aligned}
$$

where the second inequality follows from (9), the third follows because $0<\lambda_{N} \bar{L} \leq 1$, and the fourth because $0<\lambda_{\min } \leq \lambda_{N}$.

Hence, the sequence $\left\{Y_{N, k}\right\}$ satisfies the conditions for the Lindeberg-Feller theorem [4] and, so,

$$
X_{t}^{N}=\sum_{k=0}^{N-1} Y_{N, k} \stackrel{\mathrm{w}}{\rightarrow} X_{t} \sim N\left(0, \rho_{t}\right) .
$$

Step 2. Now, for any $n \geq 1$ and $t_{1}, \ldots, t_{n} \geq 0$, the vector $\boldsymbol{X}_{n}^{N}=\left(X_{t_{1}}^{N}, \ldots, X_{t_{n}}^{N}\right)$ is zero mean. For any real-valued $n$-tuple $\boldsymbol{\gamma}=\left(\gamma_{1}, \ldots, \gamma_{n}\right)$, the procedure used in Step 1 can be applied to represent the random variable $\boldsymbol{\gamma} \cdot \boldsymbol{X}_{n}^{N}$ as a sum of $N$ independent random variables, which converges to a Gaussian random variable. Furthermore, the variance of $\boldsymbol{\gamma} \cdot \boldsymbol{X}_{n}^{N}$ converges to that of $\boldsymbol{\gamma} \cdot \boldsymbol{X}_{n}$, where $\boldsymbol{X}_{n}=\left(X_{t_{1}}, \ldots, X_{t_{n}}\right)$ is an $n$-dimensional zero-mean Gaussian vector whose covariance matrix components are $\mathrm{E}\left[X_{t_{i}} X_{t_{j}}\right]=\rho_{t_{i}, t_{j}}$. Then, by the Cramér-Wold device [4], $\left(X_{t_{1}}^{N}, \ldots, X_{t_{n}}^{N}\right) \stackrel{\mathrm{w}}{\rightarrow}\left(X_{t_{1}}, \ldots, X_{t_{n}}\right)$, so the finite-dimensional distributions converge as claimed.

Step 3. We now extend the result to weak convergence of measures on $D[0, T]$. The following upper bound on the second moment of $X_{t}^{N}$ is used to carry out this step:

$$
\begin{aligned}
\mathrm{E}\left[\left(X_{t}^{N}\right)^{2}\right] & =\frac{1}{\lambda_{N} \bar{L} N} \sum_{k=0}^{N-1} \mathrm{E}\left[S_{k}^{N}(t)^{2}\right]=\frac{\lfloor N t\rfloor}{N}+2 \sum_{j=1}^{\lfloor t\rfloor}\left(\frac{\lfloor N t\rfloor}{N}-j\right) g(j+1) \\
& \leq t+2 \sum_{j=1}^{\lfloor t\rfloor}(t-j) g(j+1) \leq t+2 \sum_{j=1}^{\lfloor t\rfloor}(t-j) \\
& \leq t(1+2 T):=F(t),
\end{aligned}
$$

where the first equality follows from independence of phases and $\mathrm{E}\left[S_{k}^{N}(t)\right]=0$, the second equality follows from (10), the second inequality follows from the fact that $g(l) \leq g(1)=1$ for $l \geq 1$, and the third inequality follows from the fact that $\lfloor t\rfloor \leq T$.

Now consider any $r, s$, and $t$ such that $0 \leq r<s<t \leq T$. Also, assume that $\lfloor N s\rfloor-\lfloor N r\rfloor$ and $\lfloor N t\rfloor-\lfloor N s\rfloor$ are both nonzero, for otherwise one of the increments $X_{s}^{N}-X_{r}^{N}$ or $X_{t}^{N}-X_{s}^{N}$ is identically zero. It follows that

$$
\lfloor N s\rfloor-\lfloor N r\rfloor \leq N(t-r) \quad \text { and } \quad\lfloor N t\rfloor-\lfloor N s\rfloor \leq N(t-r) .
$$


On the one hand, if $0 \leq\lfloor N t\rfloor-\lfloor N r\rfloor \leq N$ then the increments $X_{s}^{N}-X_{r}^{N}$ and $X_{t}^{N}-X_{s}^{N}$ are independent since they correspond to arrivals in different phases. So, in this case, if $\mu>0$ then

$$
\begin{aligned}
\mathrm{P}\left[\left|X_{s}^{N}-X_{r}^{N}\right| \wedge\left|X_{t}^{N}-X_{s}^{N}\right| \geq \mu\right] & =\mathrm{P}\left[\left|X_{s}^{N}-X_{r}^{N}\right| \geq \mu\right] \mathrm{P}\left[\left|X_{t}^{N}-X_{s}^{N}\right| \geq \mu\right] \\
& \leq \frac{1}{\mu^{2}} \mathrm{E}\left[\left|X_{s}^{N}-X_{r}^{N}\right|^{2}\right] \frac{1}{\mu^{2}} \mathrm{E}\left[\left|X_{t}^{N}-X_{s}^{N}\right|^{2}\right] \\
& \leq \frac{1}{\mu^{4}} F\left(\frac{\lfloor N s\rfloor-\lfloor N r\rfloor}{N}\right) F\left(\frac{\lfloor N t\rfloor-\lfloor N s\rfloor}{N}\right) \\
& \leq \frac{1}{\mu^{4}} F(t-r)^{2} \\
& =\frac{1}{\mu^{4}}(F(t)-F(r))^{2},
\end{aligned}
$$

where the first inequality follows from Chebyshev's inequality, the second follows from the stationarity of the increments of $X_{i / N}^{N}$ and from (11), the third follows from (12) and from $F(\cdot)$ being increasing, and the final equality follows from the linearity of $F(\cdot)$. We also use ' $\wedge$ ' to denote the minimum function.

On the other hand, if $\lfloor N t\rfloor-\lfloor N r\rfloor>N$, the increments are not independent. In this case, if $\mu>0$ then

$$
\begin{aligned}
\mathrm{P}\left[\left|X_{s}^{N}-X_{r}^{N}\right| \wedge\left|X_{t}^{N}-X_{s}^{N}\right|\right. & \geq \mu] \leq \mathrm{P}\left[\left|X_{s}^{N}-X_{r}^{N}\right| \geq \mu\right] \leq \frac{1}{\mu^{2}} \mathrm{E}\left[\left|X_{s}^{N}-X_{r}^{N}\right|^{2}\right] \\
& \leq \frac{1}{\mu^{2}} F\left(\frac{\lfloor N s\rfloor-\lfloor N r\rfloor}{N}\right) \leq \frac{1}{\mu^{2}} F(t-r) \\
& \leq \frac{1}{\mu^{2}} F(t-r)^{2} \\
& =\frac{1}{\mu^{2}}(F(t)-F(r))^{2},
\end{aligned}
$$

where the first inequality follows from the fact that the probability of the intersection of two events is smaller than the probability of either one of them. Then, the second inequality follows from Chebyshev's inequality, the third follows from the stationarity of the increments of $X_{i / N}^{N}$ and from (11), the fourth follows from (12) and $F(\cdot)$ being increasing, the fifth follows from the fact that $F(t-r)$ is greater than 1 , and the equality follows from the linearity of $F(\cdot)$.

Hence, for any $r, s$, and $t$ such that the above assumptions hold,

$$
\mathrm{P}\left[\left|X_{s}^{N}-X_{r}^{N}\right| \wedge\left|X_{t}^{N}-X_{s}^{N}\right| \geq \mu\right] \leq \frac{1}{\mu^{2} \wedge \mu^{4}}(F(t)-F(r))^{2} .
$$

Also, for any $\delta>0$, the increment $X_{T}-X_{T-\delta} \sim \mathcal{N}\left(0, \rho_{\delta}\right)$ and, therefore, $X_{T}-X_{T-\delta} \stackrel{\mathrm{w}}{\rightarrow} 0$ as $\delta \rightarrow 0$.

Then, by [2, Theorem 13.5], $X_{t}^{N} \stackrel{\mathrm{w}}{\rightarrow} X_{t}$, where $X_{t}$ is a stationary-increment Gaussian process with variance as indicated by (6). The theorem has thus been proved.

\section{Properties of the limit process}

In this section, we discuss three properties of the limit process $X_{t}$ : in Section 4.1, we describe its derivative; in Section 4.2, its distribution between integer time intervals; and, in Section 4.3, a law of large numbers. 


\subsection{The derivative of the limit process}

Although the derivative process $\dot{X}_{t}$ exists only as a generalized Gaussian random process, its autocorrelation function gives insight into $X_{t}$ :

$$
\mathrm{E}\left[\dot{X}_{t_{1}} \dot{X}_{t_{2}}\right]=\frac{\partial^{2} \rho_{t_{1}, t_{2}}}{\partial t_{1} \partial t_{2}}=-\frac{1}{2} \frac{\partial^{2} \rho_{\left|t_{1}-t_{2}\right|}}{\partial t_{1} \partial t_{2}}=\sum_{n=-\infty}^{\infty} g(|n|+1) \delta\left(t_{1}-t_{2}-n\right),
$$

where the second equality follows from the stationarity of the increments of $X_{t}$ and $\delta(\cdot)$ is the Dirac delta function. Thus, formally, $\dot{X}_{t_{1}}$ is independent of $\dot{X}_{t_{2}}$ unless $t_{1}-t_{2}$ is an integer, and $\mathrm{E}\left[\dot{X}_{t_{1}} \dot{X}_{t_{1}+n}\right]$ is proportional to $g(|n|+1)$. This property mirrors the properties of the original process, specifically the autocorrelation function (1). It also is consistent with the fact that $\left\{X_{t+\alpha}-X_{t}\right\}_{0 \leq t \leq 1}$ is a standard Brownian motion for fixed $\alpha \geq 0$.

\subsection{The distribution of the process between integer time intervals}

The process $\left(X_{t}\right)_{t \in \mathbb{R}^{+}}$can be viewed as an interpolation of $\left(X_{n}\right)_{n \in \mathbb{N}^{+}}$, where Brownian bridges are used for the interpolation. A Brownian bridge is a stationary-increment, zero-mean, almost-surely-continuous Gaussian process $\left\{B_{t}\right\}_{0 \leq t \leq 1}$ with covariance function $\mathrm{E}\left[B_{s} B_{t}\right]=$ $\min \{s, t\}(1-\max \{s, t\})$. See Karatzas and Shreve [6] for more details. This characterization of $X_{t}$ is helpful in obtaining bounds on the overflow probability of the limit queueing system since [6], if $B$ is a Brownian bridge, then, for $\alpha+\beta \geq 0$ and $\beta>0$,

$$
\mathrm{P}\left[\max _{0 \leq t \leq 1}\left\{B_{t}-\alpha t\right\} \leq \beta\right]=1-\exp (-2 \beta(\alpha+\beta)) .
$$

Define, for $j \in \mathbb{N}$ and for $0 \leq t \leq 1$,

$$
\eta_{t}^{j}:=X_{t+j}-\left[(1-t) X_{j}+t X_{j+1}\right]
$$

Theorem 3. For each $j \in \mathbb{N}, \eta^{j}=\left\{\eta_{t}^{j}\right\}_{0 \leq t \leq 1}$ is a Brownian bridge and is independent of $\left(X_{n}\right)_{n \in \mathbb{N}}$. Furthermore, $\operatorname{cov}\left(\eta_{s}^{j}, \eta_{t}^{j+n}\right)=g(|n|+1) \min \{s, t\}(1-\max \{s, t\})$.

Proof. Since $\left(X_{t}\right)_{t \in \mathbb{R}}$ is a zero-mean Gaussian process, so is $\eta^{j}$. Hence, the mean and covariance function of $\eta^{j}$ specify its distribution completely. Let $j \in \mathbb{N}$ and suppose that $0 \leq s \leq t \leq 1$. Then,

$$
\begin{aligned}
\operatorname{cov}\left(\eta_{s}^{j}, \eta_{t}^{j}\right)= & \rho_{s+j, t+j}-(1-t) \rho_{s+j, j}-t \rho_{s+j, j+1}-(1-s) \rho_{j, t+j}+(1-s)(1-t) \rho_{j} \\
& +(1-s) t \rho_{j, j+1}-s \rho_{j+1, j+t}+s(1-t) \rho_{j+1, j}+s t \rho_{j+1} \\
= & s-s t
\end{aligned}
$$

using (7) and simple algebraic manipulation. Similarly, $\operatorname{cov}\left(\eta_{s}^{j}, \eta_{t}^{j}\right)=t-s t$ if $t<s$. Therefore, $\operatorname{cov}\left(\eta_{s}^{j}, \eta_{t}^{j}\right)=\min \{s, t\}(1-\max \{s, t\})$ and, hence, $\eta^{j}$ is a Brownian bridge.

Now, to show the claimed independence, it suffices to check that $\operatorname{cov}\left(\eta_{t}^{j}, X_{n}\right)=0$ for any $j, n \in \mathbb{N}$ and $0 \leq t \leq 1$, since $\left(X_{n}\right)_{n \in \mathbb{N}}$ and $\left(\eta^{j}\right)_{j \in \mathbb{N}}$ are jointly Gaussian. Indeed,

$$
\operatorname{cov}\left(\eta_{t}^{j}, X_{n}\right)=\rho_{t+j, n}-(1-t) \rho_{j, n}-t \rho_{j+1, n}=0,
$$

using (7) and simple algebraic manipulation. Therefore, $\eta^{j}$ is independent of $t\left(X_{n}\right)_{n \in \mathbb{N}}$. 
Let $j \in \mathbb{N}, n \in \mathbb{Z}$, and $0 \leq s, t \leq 1$. Then

$$
\begin{aligned}
\operatorname{cov}\left(\eta_{s}^{j}, \eta_{t}^{j+n}\right)= & \operatorname{cov}\left(\eta_{s}^{j}, X_{t+j+n}-\left[(1-t) X_{j+n}+t X_{j+n+1}\right]\right) \\
= & \rho_{s+j, t+j+n}-(1-s) \rho_{j, t+j+n}-s \rho_{j+1, t+j+n} \\
= & \begin{cases}g(n+1) s(1-t), & n \geq 0 \text { and } t \geq s, \\
g(n+1) t(1-s), & n \geq 0 \text { and } t<s, \\
g(-n+1) s(1-t), & n<0 \text { and } t \geq s, \\
g(-n+1) t(1-s), & n<0 \text { and } t<s,\end{cases}
\end{aligned}
$$

where the second equality follows from independence between $\eta^{j}$ and $\left(X_{n}\right)_{n \in \mathbb{N}}$, and the third from (7) and simple algebraic manipulation.

Therefore, $\operatorname{cov}\left(\eta_{s}^{j}, \eta_{t}^{j+n}\right)=g(|n|+1) \min \{s, t\}(1-\max \{s, t\})$ and, hence, the theorem has been proved.

\subsection{A strong law of large numbers for $X$}

The following theorem shows that, under a mild condition on $\rho$, the process $X$ satisfies a strong law of large numbers (SLLN).

Theorem 4. Suppose that, for some positive constants $K$ and $\varepsilon, \rho_{t} \leq K t^{2-\varepsilon}$ for all $t$. Then $\lim _{t \rightarrow \infty} X_{t} / t=0$ almost surely.

Proof. Since $X$ and $-X$ have the same distribution, it is enough to prove that $\lim \sup _{t \rightarrow \infty}$ $X_{t} / t=0$ almost surely. The key idea of the proof is to treat $X$ at integer times, and then appeal to the fact that, over any interval of the form $[j, j+1]$ with $j$ an integer, $X$ can be written as the sum of the linear interpolation between $X_{j}$ and $X_{j+1}$ plus the Brownian bridge $\eta^{j}(t)$. Thus, for any $t \in[j, j+1]$,

$$
X_{t} \leq \max \left\{X_{j}, X_{j+1}\right\}+\mathcal{M}\left(\eta^{j}\right),
$$

where $\mathcal{M}\left(\eta^{j}\right)$ denotes the (random) maximum value of the Brownian bridge $\eta^{j}$. Let $\alpha>0$ and define $\tau_{0}=\sup \left\{t: X_{t} \geq \alpha(2 t+1)\right\}$. To complete the proof it suffices to prove that $\mathrm{P}\left[\tau_{0}<\infty\right]=1$. Define two more random times by $\tau_{1}=\sup \left\{j: X_{j} \geq \alpha j\right\}$ and $\tau_{2}=$ $\sup \left\{j: \mathcal{M}\left(\eta^{j}\right) \geq \alpha j\right\}$. If $t \geq \max \left\{\tau_{1}, \tau_{2}\right\}+1$ then $X_{\lfloor t\rfloor}<\alpha\lfloor t\rfloor \leq \alpha t, X_{\lceil t\rceil}<\alpha\lceil t\rceil \leq$ $\alpha(t+1)$, and $\mathcal{M}\left(\eta^{\lfloor t\rfloor}\right)<\alpha\lfloor t\rfloor \leq \alpha t$. Therefore, by (14), $X_{t}<\alpha(2 t+1)$ for any $t$ with $t \geq \max \left\{\tau_{1}, \tau_{2}\right\}+1$. Consequently, $\tau_{0} \leq \max \left\{\tau_{1}, \tau_{2}\right\}+1$. Since, for an integer $j, X_{j}$ is Gaussian with mean zero and variance $\rho_{j}$, we have

$$
\mathrm{P}\left[X_{j} \geq \alpha j\right] \leq \exp \left(-\frac{\alpha^{2} j^{2}}{2 \rho_{j}}\right) \leq \exp \left(-\frac{\alpha^{2} j^{\varepsilon}}{2 K}\right)
$$

and, since $\eta^{j}$ is a standard Brownian bridge for each $j, \mathrm{P}\left[\mathcal{M}\left(\eta^{j}\right) \geq \alpha j\right]=\exp \left(-2(\alpha j)^{2}\right)$, by (13). Combining the above and using a union bound yields

$$
\begin{aligned}
\mathrm{P}\left[\tau_{0} \geq t\right] & \leq \mathrm{P}\left[\tau_{1} \geq t-1\right]+\mathrm{P}\left[\tau_{2} \geq t-1\right] \\
& \leq \sum_{j=t-1}^{\infty} \mathrm{P}\left[X_{j} \geq \alpha j\right]+\sum_{j=t-1}^{\infty} \mathrm{P}\left[\mathcal{M}\left(\eta^{j}\right) \geq \alpha j\right] \\
& =\sum_{j=t-1}^{\infty}\left[\exp \left(-\frac{\alpha^{2} j^{\varepsilon}}{2 K}\right)+\exp \left(-2(\alpha j)^{2}\right)\right]
\end{aligned}
$$


meaning that $\mathrm{P}\left[\tau_{0} \geq t\right] \rightarrow 0$ as $t \rightarrow \infty$. Thus, $\mathrm{P}\left[\tau_{0}<\infty\right]=1$. In addition, (15) provides an upper bound on the tail of the distribution of $\tau_{0}$.

Let $\theta>0$ and define the random variable $Q_{0}$ by $Q_{0}:=\sup _{t \geq 0}\left\{X_{t}-\theta t\right\}$. The following corollary is an immediate consequence of Theorem 4.

Corollary 1. Under the assumption of Theorem 4, $Q_{0}$ is finite with probability 1.

\section{Convergence of the buffer distribution}

We now make the following assumption.

Assumption 1. The probability mass function $f_{L}$ of the connection lifetime $L$ satisfies $f_{L}(i) \leq$ $D i^{-(2+\varepsilon)}$ for all $i \geq 1$, for some positive constants $D$ and $\varepsilon$.

Assumption 1 ensures that $\bar{L}<\infty$ but, if $\varepsilon \leq 1$, it permits long-range dependence, meaning that $\mathrm{E}\left[L^{2}\right]$ may be infinite. The proof of the following lemma is left to the reader.

Lemma 1. Under Assumption 1, there exist constants $K_{1}, K_{2}$, and $K_{3}$ such that $F_{L}^{\mathrm{c}}(n) \leq$ $K_{1} n^{-(1+\varepsilon)}, g(l) \leq K_{2} l^{-\varepsilon}$, and $\rho_{t} \leq K_{3} t^{2-\varepsilon}$.

Theorem 5. Let Assumption 1 hold and assume that, as $N \rightarrow \infty, \lambda_{N} \bar{L} \rightarrow 1$ in such a way that $\left(1-\lambda_{N} \bar{L}\right)\left(N^{1 / 2} /\left(\lambda_{N} \bar{L}\right)^{1 / 2}\right) \rightarrow \theta$, for some constant $\theta>0$. Then

$$
\frac{Q_{0}^{N}}{\sqrt{\lambda_{N} \bar{L} N}} \stackrel{\mathrm{w}}{\rightarrow} Q_{0} .
$$

The remainder of this section constitutes a proof of Theorem 5. Equation (3) can be rewritten using (5), as follows:

$$
\begin{aligned}
Q_{0}^{N}=\sup _{m \geq 0}\left\{\tilde{A}_{m}-m\right\} & =\sup _{m \geq 0}\left\{\sqrt{\lambda_{N} \bar{L} N} X_{(m / N)}^{N}-m\left(1-\lambda_{N} \bar{L}\right)\right\} \\
& =\sqrt{\lambda_{N} \bar{L} N} \sup _{m \geq 0}\left\{X_{(m / N)}^{N}-\frac{m\left(1-\lambda_{N} \bar{L}\right)}{\sqrt{\lambda_{N} \bar{L} N}}\right\} \\
& =\sqrt{\lambda_{N} \bar{L} N} \sup _{t \geq 0}\left\{X_{t}^{N}-\frac{\lfloor N t\rfloor}{\sqrt{N}} \frac{1-\lambda_{N} \bar{L}}{\sqrt{\lambda_{N} \bar{L}}}\right\},
\end{aligned}
$$

where the final equality follows from the change of variable $t=m / N$ and the fact that the process is constant over the interval $[m / N,(m+1) / N)$.

The convergence guaranteed by Theorem 2 holds only on a finite interval of normalized time $[0, T]$, where $T$ can be arbitrarily large, but not dependent on $N$. Thus, we define the random variables

$$
\begin{aligned}
Q_{0}^{N, T} & =\sup _{0 \leq m \leq N T}\left\{\widetilde{A}_{m}-m\right\}=\sqrt{\lambda_{N} \bar{L} N} \sup _{0 \leq t \leq T}\left\{X_{t}^{N}-\frac{\lfloor N t\rfloor}{\sqrt{N}} \frac{1-\lambda_{N} \bar{L}}{\sqrt{\lambda_{N} \bar{L}}}\right\}, \\
Q_{0}^{T} & =\max _{0 \leq t \leq T}\left\{X_{t}-\theta t\right\} .
\end{aligned}
$$

Theorem 2 and the continuous mapping theorem [2] imply that, for $T$ fixed,

$$
\frac{Q_{0}^{N, T}}{\sqrt{\lambda_{N} \bar{L} N}} \stackrel{\mathrm{w}}{\rightarrow} Q_{0}^{T} .
$$


Hence, to complete the proof of Theorem 5, it suffices to establish the following two statements, for some constant $N_{0}$ :

$$
\begin{aligned}
\mathrm{P}\left[Q_{0}^{T}=Q_{0}\right] \rightarrow 1 & \text { as } T \rightarrow \infty, \\
\inf _{N_{0} \leq N<\infty} \mathrm{P}\left[Q_{0}^{N, T}=Q_{0}^{N}\right] \rightarrow 1 & \text { as } T \rightarrow \infty .
\end{aligned}
$$

Theorem 4 and its proof will be used to prove (16), and will provide a template for the proof of (17). First, select $\alpha$ so that $2 \alpha<\theta$, let $\tau_{0}$ be the random time defined in the proof of Theorem 4, and let $t_{0}$ be a constant large enough that $\theta t \geq \alpha(2 t+1)$ for $t \geq t_{0}$. Then $X_{t}-\theta t \leq 0$ for $t \geq \max \left\{\tau_{0}, t_{0}\right\}$. Consequently, for any $T \geq t_{0}, \mathrm{P}\left[Q_{0}^{T}=Q_{0}\right] \geq$ $\mathrm{P}\left[\tau_{0} \leq T\right]$ and $\mathrm{P}\left[\tau_{0} \leq T\right]$ converges to 1 as $T \rightarrow \infty$. This establishes (16). It remains to prove (17). The proof is obtained by showing that $X^{N}$ satisfies the upper-bound half of an SLLN, with the rate of convergence uniform in $N$. To do so, we will parallel the proof of Theorem 4. Equation (15) provides a bound on the tail of the distribution of $\tau_{0}$, so the idea will be to establish a similar bound for the processes $X^{N}$ that holds uniformly in $N$. The first step is to bound the process at integer times by using uniform exponential bounds on Poisson random variables, as was done using exponential bounds on the Gaussian distribution in the proof of Theorem 4. The second step is to consider bridge processes to handle the fluctuations within frames. The following lemma takes care of the first step.

Lemma 2. Given any $\alpha>0$, there exist $T_{0}, N_{0}$, and $b_{t, \alpha}$, for integers $t \geq T_{0}$, such that

$$
\begin{gathered}
\mathrm{P}\left[X_{t}^{N} \geq 3 \alpha t\right] \leq b_{t, \alpha} \quad \text { for } t \geq T_{0}, N \geq N_{0}, \\
\sum_{t=T_{0}}^{\infty} b_{t, \alpha}<\infty .
\end{gathered}
$$

Lemma 2 will be proved with the help of some other lemmas, stated below. The function $\psi(\lambda)=2 h(1+\lambda) / \lambda^{2}$, with $h(\lambda)=\lambda(\log \lambda-1)+1$ for $\lambda \in[-1,+\infty)$, plays a very useful role in the development of exponential bounds for binomial and Poisson random variables (see [10]). This function is strictly positive and strictly decreasing on the interval $[-1,+\infty)$, with $\psi(-1)=2$ and $\psi(0)=1$. Also, $\lambda \psi(\lambda)$ is strictly increasing in $\lambda$ over the interval $[-1,+\infty)$. The following lemma is a useful arrangement of the Chernoff inequality applied to Poisson random variables.

Lemma 3. Let $V$ be a Poisson random variable with mean $\mu>0$. Then

$$
\mathrm{P}[V-\mathrm{E}[V] \geq c] \leq \exp \left(-\frac{c^{2}}{2 \mu} \psi\left(\frac{c}{\mu}\right)\right) \text { for } c \geq 0 .
$$

Proof. As the log-moment-generating function of $V$ is given by $\log \mathrm{E}[\exp (s(V-\mathrm{E}[V]))]=$ $\mu\left(\mathrm{e}^{s}-1-s\right)$, the Chernoff inequality implies that, for $c \geq 0$,

$$
\begin{aligned}
\mathrm{P}[V-\mathrm{E}[V] \geq c] & \leq \min _{s \geq 0} \exp \left(-s c+\mu\left(\mathrm{e}^{s}-1-s\right)\right) \\
& =\exp \left(-\frac{c^{2}}{2 \mu} \psi\left(\frac{c}{\mu}\right)\right),
\end{aligned}
$$

which proves the lemma. 
Lemma 4. Let $V=\sum_{i=0}^{i_{0}} i V_{i}$, where $V_{i}$ is a Poisson random variable with mean $\mu_{i}, i_{0}$ is a positive integer, and the $V_{i}$ are mutually independent. Suppose that $\mathrm{E}[V]>0$, and let $\kappa=\sum_{i=0}^{i_{0}} i^{2} \mu_{i}$. Then

$$
\mathrm{P}[V-\mathrm{E}[V] \geq c] \leq \exp \left(-\frac{c^{2}}{2 \kappa} \psi\left(\frac{c i_{0}}{\kappa}\right)\right) \text { for } c \geq 0 .
$$

Furthermore, the right-hand side of (18) is monotonically increasing in $\kappa$.

Proof. If $1 \leq i \leq i_{0}$ then $\mathrm{e}^{i s}-1-i s=\sum_{k=2}^{\infty}(i s)^{k} / k ! \leq\left(i / i_{0}\right)^{2}\left(\mathrm{e}^{i_{0} s}-1-i_{0} s\right)$. Hence,

$$
\log \mathrm{E}[\exp (s(V-\mathrm{E}[V]))]=\sum_{i=1}^{i_{0}} \mu_{i}\left(\mathrm{e}^{i} s-1-i s\right) \leq \frac{\kappa}{i_{0}^{2}}\left(\mathrm{e}^{i_{0} s}-1-i_{0} s\right)
$$

Thus, the log-moment-generating function for the centered compound Poisson random variable $V-\mathrm{E}[V]$ is less than or equal to the log-moment-generating function for $i_{0}$ times a centered Poisson random variable with mean $\kappa / i_{0}^{2}$. Therefore, we can replace $c$ by $c / i_{0}$ and $\mu$ by $\kappa / i_{0}^{2}$ in the bound of Lemma 3 to yield the bound of Lemma 4. The monotonicity follows from the monotonicity of $\lambda \psi(\lambda)$.

Consider an interval of $t$ frames, for some integer $t$. Let $r(i, t)$ denote the mean number of connections contributing $i$ packets to one of the phases during the interval, for $1 \leq i \leq t$. Such connections (i) either start or end during the interval, but not both; (ii) both start and end during the interval; or (iii) start before the interval and end after the interval. Accounting for the mean number of each of these types of connections yields

$$
r(i, t)=\lambda_{N}\left[2 \sum_{j=i+1}^{\infty} f_{L}(j)+(t-i+1) f_{L}(i)+\mathbf{1}_{\{i=t\}} \sum_{j=t+2}^{\infty}(j-t-1) f_{L}(j)\right]
$$

Comparisons with integrals show that Assumption 1 implies the following bounds, for some constants $D_{1}, D_{2}, D_{3}$, and $D_{4}$ :

$$
\begin{aligned}
r(i, t) & \leq \begin{cases}D_{1} t i^{-(2+\varepsilon)}, & 1 \leq i<t, \\
D_{2} t^{-\varepsilon}, & i=t,\end{cases} \\
\sum_{j=\left\lfloor t^{\varepsilon}\right\rfloor}^{t} r(j, t) & \leq D_{3} t^{-\varepsilon}, \\
\sum_{j=1}^{t} i^{2} r(i, t) & \leq D_{4} t^{2-\varepsilon} .
\end{aligned}
$$

Connections contributing one or more packets during the interval of frames 0 to $t-1$ are classified into two types, as follows. A connection that generates between one and $\left\lfloor t^{\varepsilon}\right\rfloor$ packets during the interval is called a type-one connection. A connection that generates between $\left\lfloor t^{\varepsilon}\right\rfloor+1$ and $t$ packets during the interval is called a type-two connection. We can then write

$$
X_{t}^{N}=\frac{Z_{1}-\mathrm{E}\left[Z_{1}\right]+Z_{2}-\mathrm{E}\left[Z_{2}\right]}{\sqrt{\lambda_{N} L N}},
$$


where $Z_{i}$ is the total number of packets contributed by type- $i$ connections during the interval of $t$ frames. Since $\lambda_{N} \bar{L} \rightarrow 1$ as $N \rightarrow \infty$, it can be assumed that $N_{0}$ is chosen large enough that $3\left(\lambda_{N} \bar{L}\right)^{1 / 2} \geq 2$ for all $N \geq N_{0}$. Then

$$
\begin{aligned}
\mathrm{P}\left[X_{t}^{N} \geq 3 \alpha t\right] & \leq \mathrm{P}\left[Z_{1}-\mathrm{E}\left[Z_{1}\right]+Z_{2}-\mathrm{E}\left[Z_{2}\right] \geq 2 \alpha \sqrt{N} t\right] \\
& \leq \mathrm{P}\left[Z_{1}-\mathrm{E}\left[Z_{1}\right] \geq \alpha \sqrt{N} t\right]+\mathrm{P}\left[Z_{2}-\mathrm{E}\left[Z_{2}\right] \geq \alpha \sqrt{N} t\right] .
\end{aligned}
$$

The random variables $Z_{1}$ and $Z_{2}$ have compound Poisson distributions, and can be expressed as

$$
\begin{aligned}
& Z_{1} \stackrel{\mathrm{D}}{=} \sum_{i=1}^{\left\lfloor t^{\varepsilon}\right\rfloor} i \text { Poisson }(N r(i, t)), \\
& Z_{2} \stackrel{\mathrm{D}}{=} \sum_{i=\left\lfloor t^{\varepsilon}\right\rfloor+1}^{t} i \text { Poisson }(N r(i, t)) .
\end{aligned}
$$

To derive a bound for $Z_{1}$, let $\kappa_{0}=N D_{4} t^{2-\varepsilon}$ and observe that $N \sum_{i=1}^{\left\lfloor t^{\varepsilon}\right\rfloor} i^{2} r(i, t) \leq$ $N \sum_{i=1}^{t} i^{2} r(i, t) \leq \kappa_{0}$. Lemma 4 then yields that, for $N \geq N_{0}$ (where $N_{0}$ is to be specified),

$$
\begin{aligned}
\mathrm{P}\left[Z_{1}-\mathrm{E}\left[Z_{1}\right] \geq \alpha t \sqrt{N}\right] & \leq \exp \left(-\frac{\alpha^{2} t^{2} N}{2 \kappa_{0}} \psi\left(\frac{\alpha t \sqrt{N} t^{1-\varepsilon}}{\kappa_{0}}\right)\right) \\
& \leq \exp \left(\frac{-\alpha^{2} t^{\varepsilon}}{2 D_{4}} \psi\left(\frac{\alpha}{D_{4} \sqrt{N_{0}}}\right)\right) .
\end{aligned}
$$

To derive a bound for $Z_{2}$, select a constant $\gamma$ with $\gamma \geq$ e. Two cases will be considered. In the first case, we assume that $\alpha N^{1 / 2} \leq \gamma N D_{3} t^{-\varepsilon}$ or, equivalently, that $N^{1 / 2} \geq \alpha t^{\varepsilon} /\left(\gamma D_{3}\right)$. Inequality (19) shows that the mean number of connections that contribute at least one packet to $Z_{2}$ is less than or equal to $N D_{3} t^{-\varepsilon}$. Therefore, the log-moment-generating function for $Z_{2}-\mathrm{E}\left[Z_{2}\right]$ is less than or equal to the log-moment-generating function of $t$ times a Poisson random variable with mean $N D_{3} t^{-\varepsilon}$. Hence, for $N^{1 / 2} \geq \alpha t^{\varepsilon} /\left(\gamma D_{3}\right)$,

$\mathrm{P}\left[Z_{2}-\mathrm{E}\left[Z_{2}\right] \geq \alpha t \sqrt{N}\right] \leq$ the Chernoff bound for $\mathrm{P}\left[\operatorname{Poisson}\left(N D_{3} t^{-\varepsilon}\right)-N D_{3} t^{-\varepsilon} \geq \alpha \sqrt{N}\right]$

$$
\begin{aligned}
& =\exp \left(-\frac{\alpha^{2} N}{2 N D_{3} t^{-\varepsilon}} \psi\left(\frac{\alpha \sqrt{N}}{N D_{3} t^{-\varepsilon}}\right)\right) \\
& \leq \exp \left(-\frac{\alpha^{2} t^{\varepsilon}}{2 D_{3}} \psi(\gamma)\right) .
\end{aligned}
$$

In the second case, we assume that $\alpha N^{1 / 2} \geq \gamma N D_{3} t^{-\varepsilon}$ and $N \geq N_{0}$, where, again, the constant $N_{0}$ is yet to be determined. The following lemma is based on the well-known idea of bounding the tail of a Poisson distribution by a geometric series.

Lemma 5. Let $V$ be a Poisson random variable with mean $\mu$ and let $c$ be a constant such that $c \geq \mu \mathrm{e}$ and $c \geq 1$. Then $\mathrm{P}[V \geq c] \leq(\mu \mathrm{e} / c)^{c}$.

Proof. If $\mu \mathrm{e}=c$ then the lemma is trivial, so suppose that $\mu \mathrm{e}<c$. Then

$$
\mathrm{P}[V \geq c]=\sum_{i=\lceil c\rceil}^{\infty} \frac{\exp (-\mu) \mu^{i}}{i !} \leq \frac{\exp (-\mu) \mu^{\lceil c\rceil}}{\lceil c\rceil !} \sum_{i=\lceil c\rceil}^{\infty}\left(\frac{\mu}{c}\right)^{i-\lceil c\rceil}=\frac{c}{c-\mu} \frac{\exp (-\mu) \mu^{\lceil c\rceil}}{\lceil c\rceil !}
$$


However, $\exp (-\mu) \leq 1$; by Stirling's formula $\lceil c\rceil ! \geq(\lceil c\rceil / \mathrm{e})^{\lceil c\rceil}(2 \pi)^{1 / 2}$; and $c /(c-\mu) \leq$ $\mathrm{e} /(\mathrm{e}-1) \leq(2 \pi)^{1 / 2}$, so $\mathrm{P}[V \geq c] \leq(\mu \mathrm{e} /\lceil c\rceil)^{\lceil c\rceil} \leq(\mu \mathrm{e} / c)^{c}$, as required.

The variable $Z_{2}$ is less than what it would be if additional packets were counted in such a way that every type-two connection generated $t$ packets. Again, using that the mean number of such connections is less than or equal to $\lambda=N D_{3} t^{-\varepsilon}$, and using Lemma 5, yields

$$
\begin{aligned}
\mathrm{P}\left[Z_{2}-\mathrm{E}\left[Z_{2}\right] \geq \alpha t \sqrt{N}\right] & \leq \mathrm{P}\left[Z_{2} \geq \alpha t \sqrt{N}\right] \\
& \leq \mathrm{P}\left[\operatorname{Poisson}\left(N D_{3} t^{-\varepsilon}\right) \geq \alpha \sqrt{N}\right] \\
& \leq\left(\frac{\sqrt{N} D_{3} t^{-\varepsilon} \mathrm{e}}{\alpha}\right)^{\alpha \sqrt{N}} .
\end{aligned}
$$

The logarithm of the right-hand side of (23) is a convex function of $N^{1 / 2}$, so it is maximized over the relevant range of $N^{1 / 2}$, namely $N_{0}^{1 / 2} \leq N^{1 / 2} \leq \alpha t^{\varepsilon} /\left(\gamma D_{3}\right)$, at one of the endpoints. Thus, for $N$ in this range,

$$
\mathrm{P}\left[Z_{2}-\mathrm{E}\left[Z_{2}\right] \geq \alpha t \sqrt{N}\right] \leq \max \left\{\left(\frac{\mathrm{e}}{\gamma}\right)^{-\alpha^{2} t^{\varepsilon} / \gamma D_{3}},\left(\frac{\sqrt{N_{0}} D_{3} t^{-\varepsilon} \mathrm{e}}{\alpha}\right)^{\alpha \sqrt{N_{0}}}\right\} .
$$

Patching together the bound (22) for the first case and the bound (24) for the second case yields that, for all $N \geq N_{0}$ and $T \geq T_{0}$,

$$
\begin{aligned}
& \mathrm{P}\left[Z_{2}-\mathrm{E}\left[Z_{2}\right] \geq \alpha t \sqrt{N}\right] \\
& \quad \leq \max \left\{\exp \left(-\frac{\alpha^{2} t^{\varepsilon}}{2 D_{3}} \psi(\gamma)\right),\left(\frac{\mathrm{e}}{\gamma}\right)^{-\alpha^{2} t^{\varepsilon} / \gamma D_{3}},\left(\frac{\sqrt{N_{0}} D_{3} t^{-\varepsilon} \mathrm{e}}{\alpha}\right)^{\alpha \sqrt{N_{0}}}\right\} .
\end{aligned}
$$

To ensure that the right-hand side of (25) is summable in $t$, we require $N_{0}$ to be large enough that $\varepsilon \alpha N_{0}^{1 / 2}>1$. Combining (20), (21), and (25) completes the proof of Lemma 2.

The second step of the proof of (17) is to bound the fluctuations of $X^{N}$ within one frame by using a uniform exponential bound on discrete versions of the Brownian bridge, similar to the exact distribution for $\mathcal{M}\left(\eta^{j}\right)$ used in the proof of Theorem 4. Given an integer $j \geq 0$, and $N \geq 1$, let $\eta^{N, j}$ denote the bridge process, defined in terms of $X^{N}$ the same way the Brownian bridge $\eta^{j}$ was defined in terms of $X$, namely $\eta_{t}^{N, j}=X_{t+j}^{N}-\left((1-t) X_{j}^{N}+t X_{j+1}^{N}\right)$ for $0 \leq t \leq 1$.

Lemma 6. Fix a constant $s_{0}>0$. If $N$ is large enough that $N \lambda_{N} \bar{L} \geq s_{0}$, then

$$
\mathrm{P}\left[\mathcal{M}\left(\eta^{N, j}\right) \geq c\right] \leq 4 \exp \left(\frac{-2 c^{2}}{\left(1+12 c / \sqrt{s_{0}}\right)}\right) .
$$

Proof. The process $\eta^{N, j}$ has the same distribution as a standard Poisson bridge process (for the same mean number of points $s=N \lambda_{N} \bar{L}$ ) on the interval [0,1], sampled at times of the form $j / M, 0 \leq j \leq M$. The process in between such points is nonincreasing, so that $\eta^{N, j}$ is maximized at a point of this form. Thus, $\mathcal{M}$ is stochastically smaller than the maximum of the standard Poisson bridge. By Inequality 7 of [10, p. 575] (with $b=\frac{1}{2}$ in that expression), $\mathrm{P}\left[\mathcal{M}\left(\eta^{N, j}\right) \geq c\right] \leq 4 \exp \left(-2 c^{2} \psi\left(4 c / s^{1 / 2}\right)\right)$, where $s=N \lambda_{N} \bar{L}$ is the mean number of packets arriving in the interval (or points, as above). The proof is completed by applying the bound $\psi(u) \leq 1 /(1+u / 3)$ for $u \geq-1$, also found in [10]. 
Lemmas 2 and 6 and the method of proof of Theorem 4 together imply (17), which completes the proof of Theorem 5 .

\section{On the distribution of buffer length for the limit process}

The authors have not found a feasible way of exactly computing the overflow probability $\mathrm{P}\left[Q_{0} \geq \beta\right]$, but lower bounds can be obtained for it, for $\beta>0$. In this section, we present three different lower bounds, and also describe the most likely path that the process will take to overflow.

\subsection{Three lower bounds}

Here, we present three different lower bounds. Let $A \subset \mathbb{R}_{+}$and let $\beta>0$. Then

$$
\mathrm{P}\left[\sup _{t \geq 0}\left\{X_{t}-\theta t\right\} \geq \beta\right] \geq \mathrm{P}\left[\sup _{t \in A}\left\{X_{t}-\theta t\right\} \geq \beta\right]:=P_{\theta}^{\mathrm{lb}}(\beta) .
$$

This lower bound is considered for three different choices of $A$.

6.1.1. $A=[0,1]$. In this case, since $X_{t}$ is a Wiener process on $[0,1]$, the first lower bound $P_{\theta}^{\mathrm{lb}_{1}}(\beta)$ is obtained as follows:

$$
\begin{aligned}
P_{\theta}^{\mathrm{lb}_{1}}(\beta) & =\int_{-\infty}^{\beta+\theta} \mathrm{P}\left[\max _{0 \leq t \leq 1}\left\{X_{t}-\theta t\right\} \geq \beta \mid X_{1}=u\right] \mathrm{P}\left[X_{1} \in[u, u+\mathrm{d} u)\right]+\mathrm{P}\left[X_{1} \geq \beta+\theta\right] \\
& =\int_{-\infty}^{\beta+\theta} \exp (-2 \beta(\beta+\theta-u)) \mathrm{P}\left[X_{1} \in[u, u+\mathrm{d} u)\right]+Q(\beta+\theta) \\
& =\exp (-2 \theta \beta)[1-Q(\theta-\beta)]+Q(\beta+\theta)
\end{aligned}
$$

where $Q(\cdot)$ denotes the complementary distribution function of a Gaussian random variable with zero mean and unit variance. The second equality follows because conditioning on the right endpoint of $A(t=1)$ yields a Brownian bridge [2, p. 101], and (13) can be used.

6.1.2. $A=\{t\}$. In this case, we recover a well-known lower bound (i.e. the basic approximation in Addie et al. [1] and Norros [8]), since $X_{t} \sim N\left(0, \rho_{t}\right)$ with $\rho_{t}$ as defined in (6). Hence, $P_{\theta}^{\mathrm{lb}_{2}}(\beta, t):=\mathrm{P}\left[X_{t}-\theta t \geq \beta\right]=Q\left((\theta t+\beta) / \rho_{t}^{1 / 2}\right)$. This lower bound can be further refined by taking the supremum over $t \geq 0$, to obtain the second lower bound

$$
P_{\theta}^{\mathrm{lb}_{2}}(\beta)=\sup _{t \geq 0} P_{\theta}^{\mathrm{lb}_{2}}(\beta, t)= \begin{cases}1, & \beta=0, \\ Q\left(\min _{t \geq 0} \frac{\theta t+\beta}{\rho_{t}^{1 / 2}}\right), & \beta>0 .\end{cases}
$$

Since $\rho_{t}$ is a piecewise linear function, $P_{\theta}^{\mathrm{lb}_{2}}(\beta)$ can be easily computed by numerical means.

$P_{\theta}^{\mathrm{lb}_{2}}(\beta)$ is expected to be a tighter bound than $P_{\theta}^{\mathrm{lb}_{1}}(\beta)$ for large values of $\beta$ since, in this case, overflow is most likely to occur over larger time-scales than $t=1$, making $P_{\theta}^{\mathrm{lb}_{1}}(\beta) \mathrm{a}$ looser bound. We denote by $t_{\mathrm{lb}_{2}}^{*}$ the optimizing value of $t$ in (27).

6.1.3. $A=[T, T+1]$, for nonnegative integer $T$. In this case, the property presented in Theorem 3, namely that $X$ is a Brownian bridge between integer time intervals, independent of the values it takes at the endpoints, becomes very useful. 
For any given integer $T \geq 0$, the following lower bound $P_{\theta}^{\mathrm{lb}_{3}}(\beta, T)$ can be obtained:

$$
\begin{aligned}
P_{\theta}^{\mathrm{lb}_{3}(\beta, T)} & =\mathrm{P}\left[\max _{T \leq t \leq T+1}\left\{X_{t}-\theta t\right\} \geq \beta\right] \\
= & \mathrm{P}\left[\max _{0 \leq t \leq 1}\left\{\eta_{t}-\left(\theta+X_{T}-X_{T+1}\right) t\right\} \geq \beta+\theta T-X_{T}\right] \\
= & \mathrm{P}\left[X_{T} \geq \beta+\theta T \text { or } X_{T+1} \geq \beta+\theta(T+1)\right] \\
& +\int_{-\infty}^{\beta+\theta T} \int_{-\infty}^{\beta+\theta(T+1)} \exp \left(-\frac{2}{\sigma^{2}}\left(\beta+\theta T-x_{T}\right)\left(\beta+\theta T-x_{T}+\theta+x_{T}-x_{T+1}\right)\right) \\
= & \mathrm{P}\left[\hat{X}_{T} \geq \beta \text { or } \hat{X}_{T+1} \geq \beta\right] \\
& +\int_{-\infty}^{\beta} \int_{-\infty}^{\beta} \exp \left(-\frac{2}{\sigma^{2}}\left(\beta-x_{T}\right)\left(\beta-x_{T+1}\right)\right) f_{\hat{X}}\left(x_{T}, x_{T+1}\right) \mathrm{d} x_{T+1} \mathrm{~d} x_{T} \\
= & 1-Q\left(-\frac{\beta-\hat{m}_{T}}{\rho_{T}},-\frac{\beta-\hat{m}_{T+1}}{\rho_{T+1}}, \hat{\rho}\right)+c_{0} \exp \left(c_{1}\right) Q\left(-\frac{\beta-m_{1}}{\sigma_{1}},-\frac{\beta-m_{2}}{\sigma_{2}}, \tilde{\rho}\right),
\end{aligned}
$$

where the second equality uses the definition of $\eta_{t}$ presented in Theorem 3 , and the third and fourth follow from (13), with $f_{X}$ being the joint probability density function of

$$
\left(\begin{array}{c}
X_{T} \\
X_{T+1}
\end{array}\right) \sim N\left(\left(\begin{array}{l}
0 \\
0
\end{array}\right),\left(\begin{array}{cc}
\rho_{T} & \rho_{T, T+1} \\
\rho_{T, T+1} & \rho_{T+1}
\end{array}\right)\right)
$$

in the third equality, and with $f_{\hat{X}}$ being the joint probability density function of

$$
\left(\begin{array}{c}
\hat{X}_{T} \\
\hat{X}_{T+1}
\end{array}\right) \sim N\left(\left(\begin{array}{c}
\hat{m}_{T} \\
\hat{m}_{T+1}
\end{array}\right),\left(\begin{array}{cc}
\rho_{T} & \rho_{T, T+1} \\
\rho_{T, T+1} & \rho_{T+1}
\end{array}\right)\right)
$$

in the fourth. The values of the constants in (28) can be found in Appendix A. Here $\sigma^{2}=1$ and $Q(x, y, \rho)$ is the two-dimensional Gaussian $Q$ function, and it can be numerically computed as indicated in Appendix B.

This lower bound can be further refined by taking the supremum over nonnegative integers $T$, to obtain the third desired lower bound

$$
P_{\theta}^{\mathrm{lb}_{3}}(\beta)=\sup _{T \geq 0} P_{\theta}^{\mathrm{lb}_{3}}(\beta, T) .
$$

We denote the optimizing value of $T$ in (29) by $T_{\mathrm{lb}_{3}}^{*}$.

It is clear that $P_{\theta}^{\mathrm{lb}_{3}}(\beta)$ is always a tighter bound than (i.e. greater than) both $P_{\theta}^{1 \mathrm{~b}_{1}}(\beta)$ and $P_{\theta}^{\mathrm{lb}_{2}}(\beta)$. This is because $P_{\theta}^{\mathrm{lb}_{3}}(\beta)$ is the maximum, over all integer intervals, of the probability that $X$ exceeds $\beta$ during such an interval, whereas $P_{\theta}^{\mathrm{lb}_{2}}(\beta)$ is the same for the first interval alone, and $P_{\theta}^{\mathrm{lb}_{2}}(\beta)$ is the maximum of the probability of exceeding $\beta$ only at a single time.

\subsection{The most likely path to overflow}

An interesting question concerns the path that the process takes when it overflows. It is shown in Norros [8] that, if $Z_{t}$ is a stationary-increment Gaussian process with $\mathrm{E}\left[Z_{t}\right]=0$, 
$\operatorname{cov}\left(Z_{s}, Z_{t}\right)=\rho_{s, t}$, and $\operatorname{var}\left(Z_{t}\right)=\rho_{t}$, then the most likely path that $Z_{t}$ will take to overflow a queue of size $\beta$ with constant service rate $\theta$ is given by $Z_{s}^{*}=-\left(\beta+\theta t^{*}\right) /\left(\rho_{t^{*}}\right) \rho_{-t^{*}, s}$, where $t^{*}$ minimizes $(\beta+\theta t)^{2} / \rho_{t}$. Notice that this $t^{*}$ is the same as the optimal time-scale $t_{\mathrm{lb}_{2}}^{*}$ obtained for $P_{\theta}^{\mathrm{lb}_{2}}(\beta)-$ see Norros [8] for more details.

\section{Numerical results}

In this section, we consider a specific lifetime distribution for the connections in order to present a quantitative analysis of the bounds obtained in Section 6.1. Let the lifetime of a connection have a geometric distribution with parameter $1-\alpha$ for $0 \leq \alpha<1$. Then $F_{L}^{\mathrm{c}}(l)=\alpha^{l-1}$ for $l \in\{1,2,3, \ldots\}, \bar{L}=1 /(1-\alpha)$, and $\mathrm{E}\left[L^{2}\right]<\infty$. Hence, the process is not long-range dependent. The variance function is

$$
\rho_{t}=t+2 \sum_{j=1}^{\infty} \alpha^{j}(t-j)^{+}
$$

Exact overflow probabilities are presented for two specific values of this distribution, namely $\alpha=0$ and $\alpha=1$, whereas bounds are obtained for $0<\alpha<1$. These three cases are examined in Sections 7.1, 7.2, and 7.3, respectively. We denote by $P_{\alpha, \theta}(\beta)$ the overflow probability for a particular value of $\alpha$.

\subsection{The case $\alpha=0$}

In the case $\alpha=0$, the process has no periodicity at all since connections depart after one transmission. It is straightforward to see from (30) and (7) that $X$ becomes a simple Wiener process for $t \geq 0$. Therefore [3], the overflow probability is $P_{0, \theta}(\beta)=\exp (-2 \theta \beta)$.

\subsection{The case $\alpha=1$}

In the case $\alpha=1$, the process has sample paths with periodic increments since

$$
X_{t}= \begin{cases}W_{t}, & 0 \leq t \leq 1, \\ X_{t-1}+W_{1}, & t \geq 1\end{cases}
$$

where $W$ is a Wiener process.

The overflow probability can be obtained noting that

$$
\sup _{t \geq 0}\left\{X_{t}-\theta t\right\}= \begin{cases}\infty, & W_{1}>\theta \\ \max _{0 \leq t \leq 1}\left\{W_{t}-\theta t\right\}, & W_{1} \leq \theta\end{cases}
$$

This is due to the fact that if $W_{1}>\theta$, then the periodic repetition will start above 0 and will always increase, meaning that the sample path becomes unbounded above. If, instead, $W_{1} \leq \theta$ then the process will exceed $\beta$ only if it does so in the first interval. With this in mind, this overflow probability can be calculated by following a procedure similar to the one used in (26), to obtain

$$
P_{1, \theta}(\beta)=Q(\theta)+\exp (-2 \theta \beta)[1-Q(\theta-2 \beta)]
$$




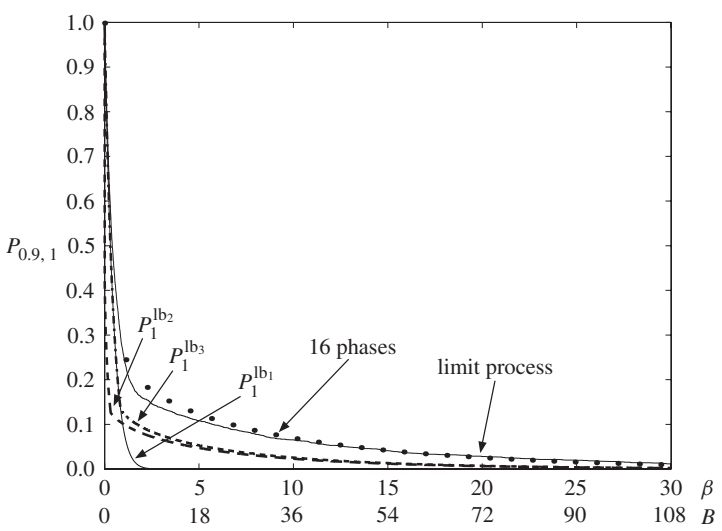

(a)

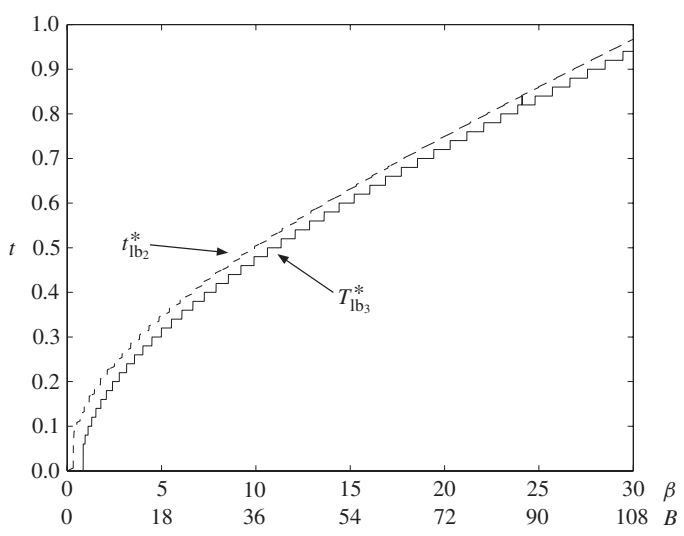

(b)

FIGURE 2: Comparisons for $\alpha=0.9, \theta=1$, and $\lambda_{N}=0.077$ 93: (a) lower bounds; (b) optimal time-scale.

\subsection{The general case $0<\alpha<1$}

This section presents the overflow probability, obtained from simulations, for both the limit process and the original process in the case that $0<\alpha<1$, and compares them to the lower bounds presented in Section 6.1. The variables $B$ and $\beta$ are related by $B=\beta\left(\lambda_{n} \bar{L} N\right)^{1 / 2}$, and Theorem 5 suggests the approximation $\mathrm{P}\left[Q_{o}^{N} \geq B\right] \approx \mathrm{P}\left[Q_{o} \geq \beta\right]$. The probabilities are plotted in Figure 2(a) with $\theta=1, \alpha=0.9$, and $\lambda_{N}=0.07793$, which, for the original system with $N=16$ phases, corresponds to a load of 0.7793 . First, it is clear that the limit process is a very good approximation to the $N=16$ process. As required, the tightest lower bound is $P_{1}^{\mathrm{lb}_{3}}$. However, $P_{1}^{\mathrm{lb}_{2}}$ is very close to $P_{1}^{\mathrm{lb}_{3}}$ for $\beta>5(B>17.66)$. Notice in Figure 2(b) that, for $\beta>0.85(B>3)$, the most likely integer time interval for the process to exceed $\beta$ is after the first interval (i.e. $T_{\mathrm{lb}_{3}}^{*} \geq 1$ ). This causes $P_{1}^{\mathrm{lb}_{1}}$, which corresponds to $T_{\mathrm{lb}_{3}}^{*}=0$, to decrease in accuracy. It can also be observed in Figure 2 that, as expected, $P_{1}^{\mathrm{lb}_{1}}$ is a tighter bound than $P_{1}^{\mathrm{lb}_{2}}$ when the frame in which the process is most likely to exceed $\beta$ is the first. While $P_{1}^{\mathrm{lb}_{3}}$ is the tightest lower bound, it is also that which requires the most computation - it requires computation of the integrals in (28). Here, the lower bounds are not a good approximation when the actual overflow probability is larger than 0.1 , but they are good for smaller overflow probabilities, which are usually the ones that are of interest to system designers. 


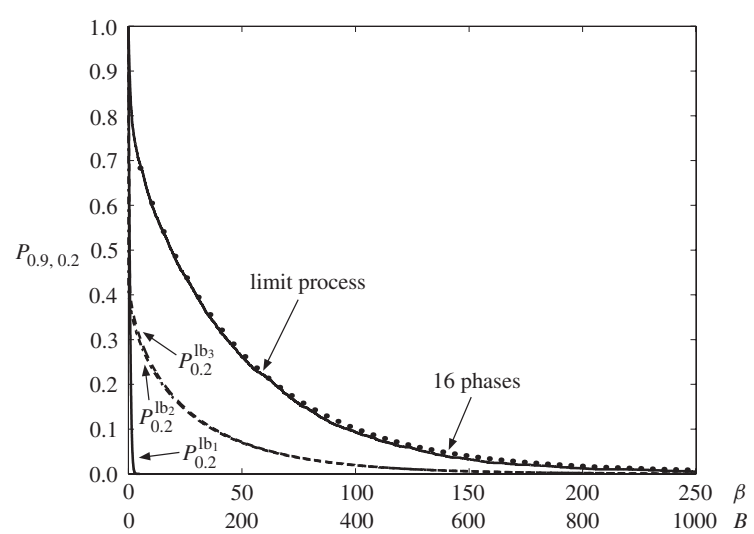

(a)

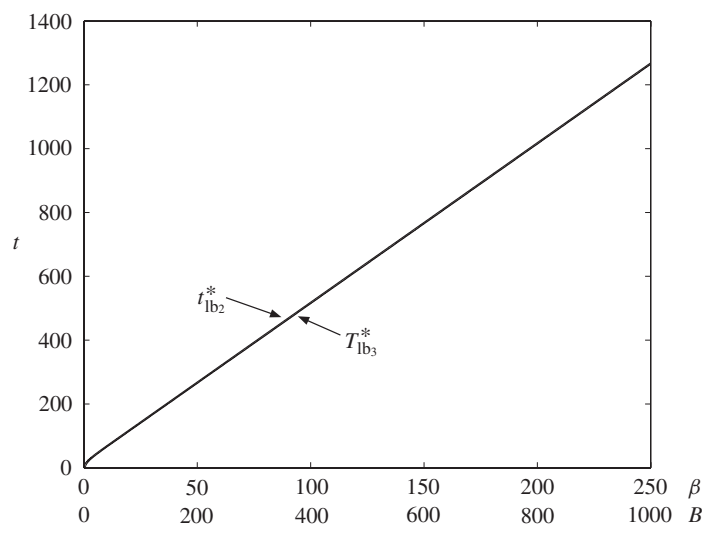

(b)

Figure 3: Comparisons for $\alpha=0.9, \theta=0.2$, and $\lambda_{N}=0.095$ 123: (a) lower bounds; (b) optimal time-scale.

Figure 2 shows a clear change in the slope of the overflow probability at $\beta=0.33(B=1.16)$ for $P_{1}^{\mathrm{lb}_{2}}$ and at $\beta=0.85(B=3)$ for $P_{1}^{\mathrm{lb}_{3}}$, where it is more likely for the process to exceed $\beta$ after the first frame. As was discussed by Pazhyannur and Fleming [9], for small values of average queueing delay (which, by Little's law, is proportional to the average queue length), the delay behaves exponentially with mean $\lambda_{1}$, while for larger values it behaves exponentially but with mean $\lambda_{2} \neq \lambda_{1}$. This 'two-scaled exponential distributions' effect, as it is called in [9], is easy to observe in the semilog plot of [9, Figure 7]. This two-scale behavior is due to correlation since, if the number of buffered packets is small, then most likely they all arrived within the space of one frame and, hence, there is no correlation between them. However, a larger number of buffered packets is most likely due to packets that arrived over multiple frames, and correlation comes into the picture. This is a characteristic of the semiperiodic behavior of the system, which is preserved in the diffusion limit. This two-scale behavior is also observed in the most likely path to overflow considered by Norros [8].

Figure 3 shows the overflow probabilities and optimal time-scales for $\theta=0.2, \alpha=0.9$, and $\lambda_{N}=0.095123$, which corresponds to a load of 0.95123 for $N=16$ phases. Again, it is clear that the limit process is already a very good approximation to the $N=16$ process. The same pattern is observed for the lower bounds. 

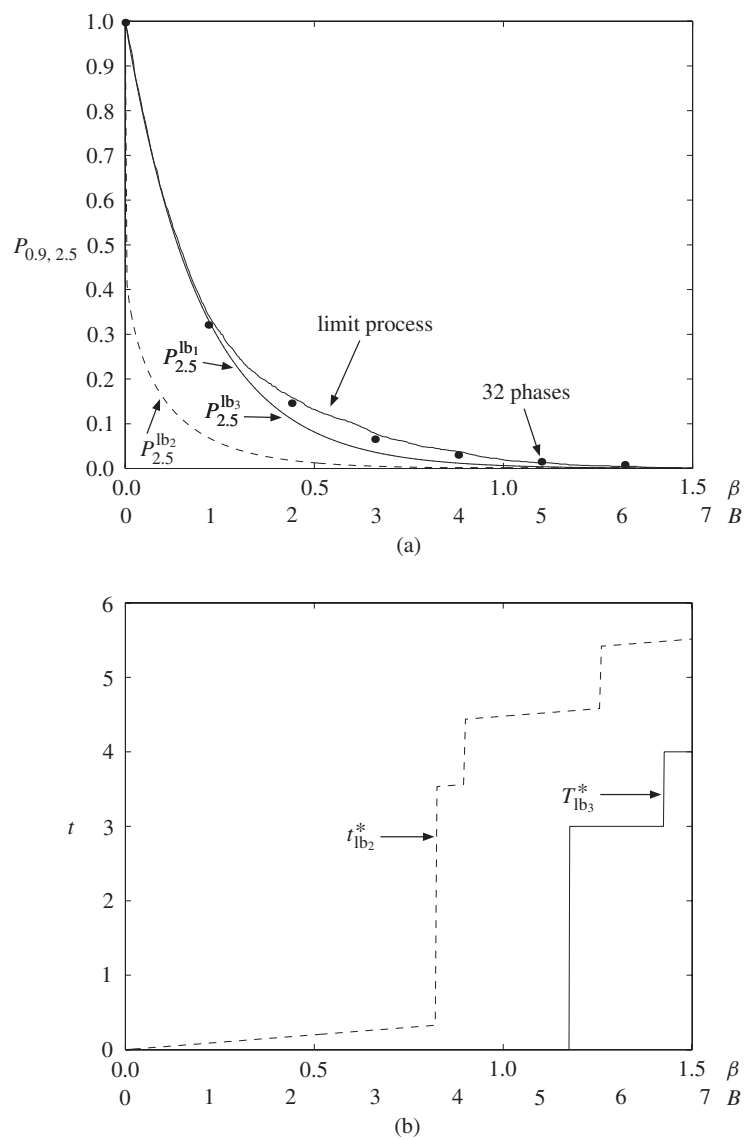

FIGURE 4: Comparisons for $\alpha=0.9, \theta=2.5$, and $\lambda_{N}=0.064505$ : (a) lower bounds; (b) optimal time-scale.

The overflow probabilities and optimal time-scales for $\theta=2.5, \alpha=0.9$, and $\lambda_{N}=$ 0.064505 , which corresponds to a load of 0.645054 for $N=32$ phases, are plotted in Figure 4 . The same conclusions are reached as for Figure 3. A plot for $N=32$ rather than for $N=16$ is shown since, for $N=16$, the overflow probability is small even for very small buffer sizes.

\subsection{The effect of mean load and correlation}

Of course, overflow probabilities increase significantly as the mean load increases (i.e. as the draining rate $\theta$ decreases). This can be observed in Figure 5, where overflow probabilities below 0.1 are achieved for $\beta>0.1$ in a lightly loaded system $(\theta=10)$, while they are achieved for $\beta>1.5$ in a heavily loaded system $(\theta=1)$. The effect of correlation on the overflow probability is a little more subtle than the effect of mean load. As discussed in Section 7.3, the impact is significant only if overflow probabilities for large enough buffers are considered, and the impact is greater for more heavily loaded systems. Figure 5 indicates a marked dependence on the correlation $\alpha$ only in the more heavily loaded system $(\theta=1)$ and for $\beta$ sufficiently large $(\beta>1)$. 


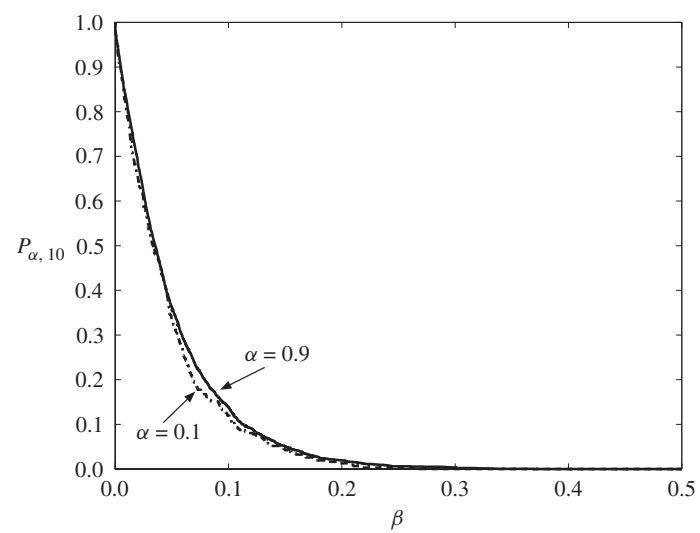

(a)

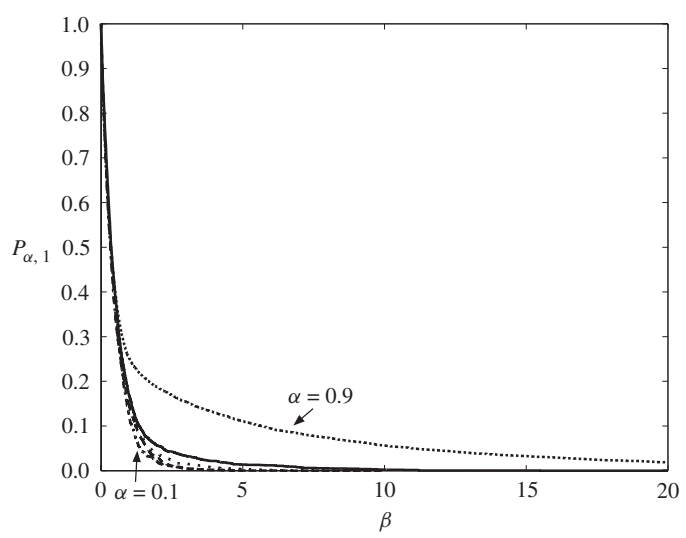

(b)

Figure 5: Overflow probability for (a) small draining rate $(\theta=10)$; (b) large draining rate $(\theta=1)$.

\section{Conclusions}

In this paper, we have presented a diffusion limit approximation of the cumulative arrival process in a discrete-time queueing system with constant bit rate connections. The diffusion scaling retains the semiperiodic behavior of the process, allowing for both short-time (within one frame) and long-time (over multiple frames) analysis in the limit. Several properties of the limit process were discussed. The limit process can be viewed as an interpolation of a stationaryincrement discrete-time Gaussian process, where interpolation is done with Brownian bridges. Under a mild condition on the tail of the connection lifetime distribution, the limit of the cumulative arrival process satisfies a strong law of large numbers. Related bounds for the actual cumulative arrival process were used to establish the limit in distribution for the scaled equilibrium buffer length.

Bounds on the overflow probability of the limit queueing system, as a function of the arrival rate and the connection lifetime distribution, were presented and some numerical results used to illustrate the approximate analysis. We found that the bounds are a good approximation when the actual probability of overflow is smaller than 0.1 . These bounds on the limit system were also compared to the overflow probability of the original system. The results of our simulations also showed that the limit approximation to the system is a very good one. 
Finally, we pointed out that the correlation effect is significant only for large buffer build up, and its effect is enhanced when the average load is high.

\section{Appendix A.}

In this appendix, we present the values of the constants that appear in the calculation of $P_{\theta}^{\mathrm{lb}_{3}}(\beta)$ in Section 6.1:

$$
\begin{aligned}
\hat{m}_{T} & =-\theta T, \quad \hat{m}_{T+1}=-\theta(T+1), \quad \hat{\rho}=\frac{\rho_{T, T+1}}{\sqrt{\rho_{T} \rho_{T+1}}}, \\
\sigma_{1}^{2} & =\frac{\sigma^{4} \rho_{T}}{\left(\sigma^{2}+2 \rho_{T, T+1}\right)^{2}-4 \rho_{T} \rho_{T+1}}, \quad \sigma_{2}^{2}=\frac{\sigma^{4} \rho_{T+1}}{\left(\sigma^{2}+2 \rho_{T, T+1}\right)^{2}-4 \rho_{T} \rho_{T+1}}, \\
\tilde{\rho} & =\frac{\rho_{T, T+1}\left(\sigma^{2}+2 \rho_{T, T+1}\right)-2 \rho_{T, T+1}}{\sigma^{2} \sqrt{\rho_{T} \rho_{T+1}}}, \quad c_{0}=\frac{\sigma^{2}}{\sqrt{\left(\sigma^{2}+2 \rho_{T, T+1}\right)^{2}-4 \rho_{T} \rho_{T+1}}}, \\
c_{1} & =\frac{2\left((\beta+\theta T)\left((\beta+(T+1) \theta)\left(\sigma^{2}+2 \rho_{T, T+1}\right)-\rho_{T+1}(\theta T+\beta)\right)-\rho_{T}(\beta+(T+1) \theta)^{2}\right)}{4 \rho_{T} \rho_{T+1}-\left(\sigma^{2}+2 \rho_{T, T+1}\right)^{2}}, \\
m_{1} & =\frac{2 \rho_{T}\left(2 \beta \rho_{T+1}-\sigma^{2}(\theta(T+1)+\beta)\right)+\left(\sigma^{2}+2 \rho_{T, T+1}\right)\left(T \theta \sigma^{2}-2 \beta \rho_{T, T+1}\right)}{4 \rho_{T} \rho_{T+1}-\left(\sigma^{2}+2 \rho_{T, T+1}\right)^{2}}, \\
m_{2} & =\frac{2 \rho_{T+1}\left(2 \beta \rho_{T}-\sigma^{2}(\theta T+\beta)\right)+\left(\sigma^{2}+2 \rho_{T, T+1}\right)\left((T+1) \theta \sigma^{2}-2 \beta \rho_{T, T+1}\right)}{4 \rho_{T} \rho_{T+1}-\left(\sigma^{2}+2 \rho_{T, T+1}\right)^{2}} .
\end{aligned}
$$

\section{Appendix B.}

Here, we present a method to simplify the integration of the two-dimensional Gaussian $Q$ function

$$
\begin{aligned}
& Q\left(x_{1}, y_{1}, \rho\right) \\
& \quad:=\frac{1}{2 \pi \sqrt{1-\rho^{2}}} \int_{x_{1}}^{\infty} \int_{y_{1}}^{\infty} \exp \left(-\frac{x^{2}+y^{2}-2 \rho x y}{2\left(1-\rho^{2}\right)}\right) \mathrm{d} x \mathrm{~d} y \\
& \quad=\frac{1}{2 \pi \sqrt{1-\rho^{2}}} \int_{0}^{\infty} \int_{0}^{\infty} \exp \left(-\frac{\left(x+x_{1}\right)^{2}+\left(y+y_{1}\right)^{2}-2 \rho\left(x+x_{1}\right)\left(y+y_{1}\right)}{2\left(1-\rho^{2}\right)}\right) \mathrm{d} x \mathrm{~d} y,
\end{aligned}
$$

where the equality follows from a simple change of variables. This can be further simplified by another change of variables that arises from the geometry of the area of interest. Define $N$, $\theta$, and $\phi_{s}$, using $j=(-1)^{1 / 2}$, by

$$
N \exp (j \theta)=\left(x+x_{1}\right)+j\left(y+y_{1}\right) \quad \text { and } \quad \phi_{s}=\arctan \left(\frac{y_{1}}{x_{1}}\right) .
$$

Changing variables from $(x, y)$ to $(N, \theta)$ and using the equality

$$
\left(x+x_{1}\right)^{2}+\left(y+y_{1}\right)^{2}-2 \rho\left(x+x_{1}\right)\left(y+y_{1}\right)=N^{2}(1-\rho \sin 2 \theta)
$$

(presented in Simon and Alouini [11]), makes $Q$ a finite double integral that can be further simplified to single integrals. 
We now present the final integrals for evaluation. The integrals are different depending on which quadrant the vector $\left(x_{1}, y_{1}\right)$ lies in.

- First quadrant:

$$
\begin{aligned}
Q\left(x_{1}, y_{1}, \rho\right)= & \frac{\sqrt{1-\rho^{2}}}{2 \pi} \int_{0}^{\phi_{s}} \frac{1}{1-\rho \sin 2 \theta} \exp \left(-\frac{y_{1}^{2}(1-\rho \sin 2 \theta)}{2\left(1-\rho^{2}\right) \sin ^{2} \theta}\right) \mathrm{d} \theta \\
& +\frac{\sqrt{1-\rho^{2}}}{2 \pi} \int_{\phi_{s}}^{\pi / 2} \frac{1}{1-\rho \sin 2 \theta} \exp \left(-\frac{x_{1}^{2}(1-\rho \sin 2 \theta)}{2\left(1-\rho^{2}\right) \cos ^{2} \theta}\right) \mathrm{d} \theta .
\end{aligned}
$$

- Second quadrant:

$$
\begin{aligned}
Q\left(x_{1}, y_{1}, \rho\right)= & \frac{\sqrt{1-\rho^{2}}}{2 \pi} \int_{0}^{\pi / 2} \frac{1}{1-\rho \sin 2 \theta} \exp \left(-\frac{y_{1}^{2}(1-\rho \sin 2 \theta)}{2\left(1-\rho^{2}\right) \sin ^{2} \theta}\right) \mathrm{d} \theta \\
& +\frac{\sqrt{1-\rho^{2}}}{2 \pi} \int_{-\pi / 2}^{\phi_{s}} \frac{1}{1-\rho \sin 2 \theta} \exp \left(-\frac{y_{1}^{2}(1-\rho \sin 2 \theta)}{2\left(1-\rho^{2}\right) \sin ^{2} \theta}\right) \mathrm{d} \theta \\
& -\frac{\sqrt{1-\rho^{2}}}{2 \pi} \int_{-\pi / 2}^{\phi_{s}} \frac{1}{1-\rho \sin 2 \theta} \exp \left(-\frac{x_{1}^{2}(1-\rho \sin 2 \theta)}{2\left(1-\rho^{2}\right) \cos ^{2} \theta}\right) \mathrm{d} \theta .
\end{aligned}
$$

- Third quadrant:

$$
\begin{aligned}
Q\left(x_{1}, y_{1}, \rho\right)= & -\frac{\sqrt{1-\rho^{2}}}{2 \pi} \int_{\phi_{s}}^{\pi / 2} \frac{1}{1-\rho \sin 2 \theta} \exp \left(-\frac{y_{1}^{2}(1-\rho \sin 2 \theta)}{2\left(1-\rho^{2}\right) \sin ^{2} \theta}\right) \mathrm{d} \theta \\
& -\frac{\sqrt{1-\rho^{2}}}{2 \pi} \int_{0}^{\phi_{s}} \frac{1}{1-\rho \sin 2 \theta} \exp \left(-\frac{x_{1}^{2}(1-\rho \sin 2 \theta)}{2\left(1-\rho^{2}\right) \cos ^{2} \theta}\right) \mathrm{d} \theta \\
& -\frac{\sqrt{1-\rho^{2}}}{2 \pi} \int_{-\pi / 2}^{0} \frac{1}{1-\rho \sin 2 \theta} \exp \left(-\frac{y_{1}^{2}(1-\rho \sin 2 \theta)}{2\left(1-\rho^{2}\right) \sin ^{2} \theta}\right) \mathrm{d} \theta \\
& -\frac{\sqrt{1-\rho^{2}}}{2 \pi} \int_{-\pi / 2}^{0} \frac{1}{1-\rho \sin 2 \theta} \exp \left(-\frac{x_{1}^{2}(1-\rho \sin 2 \theta)}{2\left(1-\rho^{2}\right) \cos ^{2} \theta}\right) \mathrm{d} \theta .
\end{aligned}
$$

- Fourth quadrant:

$$
\begin{aligned}
Q\left(x_{1}, y_{1}, \rho\right)= & \frac{\sqrt{1-\rho^{2}}}{2 \pi} \int_{\phi_{s}}^{\pi / 2} \frac{1}{1-\rho \sin 2 \theta} \exp \left(-\frac{x_{1}^{2}(1-\rho \sin 2 \theta)}{2\left(1-\rho^{2}\right) \cos ^{2} \theta}\right) \mathrm{d} \theta \\
& -\frac{\sqrt{1-\rho^{2}}}{2 \pi} \int_{\phi_{s}}^{0} \frac{1}{1-\rho \sin 2 \theta} \exp \left(-\frac{y_{1}^{2}(1-\rho \sin 2 \theta)}{2\left(1-\rho^{2}\right) \sin ^{2} \theta}\right) \mathrm{d} \theta .
\end{aligned}
$$

\section{Acknowledgement}

The authors are grateful to a referee who pointed out that weak convergence of the process $X^{N}$ on finite intervals alone is not enough to conclude convergence of the normalized buffer-length variable.

\section{References}

[1] Addie, R., Mannersalo, P. and Norros, I. (1999). Performance formulae for queues with Gaussian input. In Teletraffic Engineering in a Competitive World (Proc. Internat. Teletraffic Congress (ITC-16), Edinburgh, 1999), eds P. Key and D. Smith, Elsevier, Amsterdam, pp. 1169-1178. 
[2] Billingsley, P. (1999). Convergence of Probability Measures, 2nd edn. John Wiley, New York.

[3] Doob, J. L. (1949). Heuristic approach to the Kolmogorov-Smirnov theorems. Ann. Math. Statist. 20, $393-403$.

[4] Durrett, R. (1996). Probability: Theory and Examples, 2nd edn. Duxbury Press, Belmont, CA.

[5] HajeK, B. (1994). A queue with periodic arrivals and constant service rate. In Probability, Statistics and Optimisation: a Tribute to Peter Whittle, ed. F. P. Kelly, John Wiley, Chichester, pp. 147-157.

[6] Karatzas, I. And Shreve, S. E. (1991). Brownian Motion and Stochastic Calculus (Grad. Texts Math. 113), 2nd edn. Springer, New York.

[7] Krunz, M. M. And Makowski, A. M. (1998). Modeling video traffic using $M / G / \infty$ input processes: a compromise between Markovian and LRD models. IEEE J. Select. Areas Commun. 16, 733-748.

[8] Norros, I. (2002). Most probable path techniques for Gaussian queueing systems. In Networking 2002 (Proc. 2nd Internat. IFIP-TC6 Network. Conf., Pisa, 2002), eds E. Gregori et al., Springer, London, pp. 86-104.

[9] Pazhyannur, R. S. and Fleming, P. J. (2001). Asymptotic results for voice delay in packet networks. In Proc. IEEE 54th Vehicular Technology Conf., Vol. 3, IEEE, pp. 1861-1865.

[10] Shorack, G. R. and Wellner, J. A. (1986). Empirical Processes with Applications to Statistics. John Wiley, New York.

[11] Simon, M. K. And Alouini, M.-S. (2000). Digital Communication over Fading Channels: A Unified Approach to Performance Analysis. John Wiley, New York. 